\title{
NON-SPECIAL SCROLLS WITH GENERAL MODULI
}

\author{
ALBERTO CALABRI, CIRO CILIBERTO, FLAMINIO FLAMINI, RICK MIRANDA
}

\begin{abstract}
In this paper we study smooth, non-special scrolls $S$ of degree $d$, genus $g \geq 0$, with general moduli. In particular, we study the scheme of unisecant curves of a given degree on $S$. Our approach is mostly based on degeneration techniques.
\end{abstract}

\section{INTRODUCTION}

It is well-known that the study of vector bundles on curves is equivalent to the one of scrolls in projective space. In the present article, we will mostly take the projective point of view, together with degeneration techniques, in order to study smooth, non-special scroll surfaces of degree $d$, sectional genus $g \geq 0$, with general moduli, which are linearly normal in $\mathbb{P}^{R}, R=d-2 g+1$. However we will bridge this approach with the vector-bundle one showing how projective geometry and degenerations can be used in order to improve some known results about rank-two vector bundles and to obtain some new ones (cf. also [4]).

The first three sections of the paper basically contain some folklore, which we think will be useful for a possible reader. In $\S 2$ and 3 we fix notation and terminology and recall preliminary results on scrolls. In $\S$ 国e introduce the vector bundle setting.

If $d \geq 2 g+3+\min \{1, g-1\}$, such scrolls fill up a unique component $\mathcal{H}_{d, g}$ of the Hilbert scheme of surfaces in $\mathbb{P}^{R}$ which dominates $\mathcal{M}_{g}$ (this result is essentially contained in [2]; see [3] for an explicit statement and different proofs). Let $[S] \in \mathcal{H}_{d, g}$ be a general point, such that $S \cong \mathbb{P}(\mathcal{F})$, where $\mathcal{F}$ is a very ample rank-two vector bundle of degree $d$ on $C$, a curve of genus $g$ with general moduli, and $S$ is embedded in $\mathbb{P}^{R}$ via the global sections of $\mathcal{O}_{\mathbb{P}(\mathcal{F})}(1)$. In $\S$, we first recall that if $g \geq 2$ and $S$ is general, then $\mathcal{F}$ is stable (in case $g=1$ there is a slightly different result; cf. Theorem 5.4 and Remark 5.5. This result is contained in [2]. We give here a short, independent proof). This suggests that $\mathcal{H}_{d, g}$ plays, in the projective geometry setting, a role analogous to the one of the moduli stack of semistable rank-two vector bundles of degree $d$ over $\mathcal{M}_{g}$. We discuss in Remarks 5.7 and 5.8 a few examples showing that $\mathcal{H}_{d, g}$ contains points corresponding to unstable bundles as well as to strictly semistable products of the type $C \times \mathbb{P}^{1}$. We finish $\S$ 5y describing two constructions closely related to the ones in [3] (cf. Constructions 5.9] and 5.11) which prove that $\mathcal{H}_{d, g}$ contains smooth points corresponding to some reducible scrolls. The results in [2] also imply that $\mathcal{H}_{d, g}$ contains points corresponding to reducible scrolls. However, the ones that we need to consider in this paper are different from those in [2] and therefore we have to introduce them here. Note that in [3] one proves that $\mathcal{H}_{d, g}$ contains points corresponding to surfaces which are reducible in suitable unions of planes, thus solving an old problem posed by G. Zappa (cf. [35]). These planar degenerations however are not used here.

In $\S\left[\right.$ we consider the scheme $\operatorname{Div}_{S}^{1, m}$ parametrizing unisecant curves of given degree $m$ on $S$ (cf. Definition 6.1). By using degeneration arguments involving the above constructions,

Mathematics Subject Classification (2000): 14J26, 14D06, 14C20; (Secondary) 14H60, 14N10. Keywords: ruled surfaces; Hilbert schemes of scrolls; Moduli; embedded degenerations. The first three authors are members of G.N.S.A.G.A. at I.N.d.A.M. "Francesco Severi". 
we prove Theorem 6.9, which says that $S$ is a general ruled surface in the sense of Ghione in [11, Definition 6.1] (cf. Definition 6.6 below). Namely

(i) $\operatorname{dim}\left(\operatorname{Div}_{S}^{1, m}\right)=d_{m}:=\max \{-1,2 m-d-g+1\}$;

(ii) $\operatorname{Div}_{S}^{1, m}$ is smooth, for any $m$ such that $d_{m} \geq 0$;

(iii) $\operatorname{Div}_{S}^{1, m}$ is irreducible, for any $m$ such that $d_{m}>0$.

This, in particular, gives effective existence results for general ruled surfaces (whereas [11, Théorème 7.1] is only asymptotic, cf. Theorem 6.7 below). The idea of using degenerations to study unisecants is already present in [2], where however it is used only to prove the stability of the rank-two vector bundle determining the general point of $\mathcal{H}_{d, g}$.

In $\S 7$ we make some applications proving a few enumerative properties of $\operatorname{Div}_{S}^{1, m}$. In Theorem 7.1 we give a new and easy proof of a result of Ghione (cf. [11, Théorème 6.4 and 6.5]), which provides also an effective version of it. Then, we apply Theorem 7.1 to compute the so called index of $\operatorname{Div}_{S}^{1, m}$, thus giving a new proof of a result of Corrado Segre (cf. Proposition 7.2). In $\S 7.3$ we study the monodromy action on $\operatorname{Div}_{S}^{1, m}$ in case this is finite, proving that the monodromy acts as the full symmetric group. Finally, in $\S 7.4$ we extend the result in [16] and [27, Example 3.2], by computing the genus of the curve parametrizing those divisors in Div ${ }_{S}^{1, m}$ passing through $d_{m}-1$ general points of $S$.

This paper has to be regarded as the continuation of a project initiated with [3]. In [4] we make further application of the ideas contained in [3] and in the present paper to the BrillNoether theory of sub-line bundles of rank-two vector bundles on curves. We will devote a forthcoming article to the case of special scrolls.

\section{NotATION AND PRELIMINARIES}

In this section we will fix notation and general assumptions. As a general warning, if there is no danger of confusion, we will identify line bundles and divisors.

Let $C$ be a smooth, projective curve of genus $g \geq 0$ and let $\rho: F \rightarrow C$ be a geometrically ruled surface on $C$, i.e. $F=\mathbb{P}(\mathcal{F})$, with $\mathcal{F}$ a rank-two vector bundle on $C$. For us, a rank $r$ vector bundle is a locally free sheaf of rank $r$.

In this paper, we shall make the following:

Assumptions 2.1. With notation as above,

(1) the rank-two vector bundle $\mathcal{F}$ is of $\operatorname{degree} \operatorname{deg}(\mathcal{F}):=\operatorname{deg}(\operatorname{det}(\mathcal{F}))=d$;

(2) $h^{0}(C, \mathcal{F})=R+1$, for some $R \geq 3$;

(3) the complete linear system $\left|\mathcal{O}_{F}(1)\right|$ is base-point-free (therefore the general element is a smooth, irreducible curve on $F$ ) and the morphism

$$
\Phi: F \rightarrow \mathbb{P}^{R}
$$

induced by $\left|\mathcal{O}_{F}(1)\right|$ is birational to the image.

Definition 2.2. The surface

$$
\Phi(F):=S \subset \mathbb{P}^{R}
$$

is said to be a scroll of degree $d$ and of (sectional) genus $g$, and $S$ is called the scroll determined by the pair $(\mathcal{F}, C)$. Note that $S$ is smooth if and only if $\mathcal{F}$ is very ample. If $S$ is not smooth, then $F$ is its minimal desingularization.

For any $x \in C$, let $f_{x}:=\rho^{-1}(x) \cong \mathbb{P}^{1}$. The general fibre of $\rho$ will be denoted by $f$. For any $x \in C$, the line $l_{x}:=\Phi\left(f_{x}\right)$ is called a ruling of $S$ (the general ruling of $S$ will be denoted by $l=\Phi(f)$ ). By abusing terminology, the family $\left\{l_{x}\right\}_{x \in C}$ is also called the ruling of $S$. 
For further details on ruled surfaces, we refer the reader to [15], 18, § V], [2], 8], [9], [10], [11], [12], [13], [21], [22], 25], [29], [32] and [33].

Let $F \stackrel{\rho}{\rightarrow} C$ be as above. There is a section $i: C \hookrightarrow F$, whose image we denote by $H$, such that $\mathcal{O}_{F}(H)=\mathcal{O}_{F}(1)$. Then

$$
\operatorname{Pic}(F) \cong \mathbb{Z}\left[\mathcal{O}_{F}(H)\right] \oplus \rho^{*}(\operatorname{Pic}(C))
$$

Moreover,

$$
\operatorname{Num}(F) \cong \mathbb{Z} \oplus \mathbb{Z}
$$

generated by the classes of $H$ and $f$, satisfying $H f=1, f^{2}=0$ (cf. [18, $\S 5$, Prop. 2.3]). If $\underline{d} \in \operatorname{Div}(C)$, we denote by $\underline{d} f$ the divisor $\rho^{*}(\underline{d})$ on $F$. A similar notation will be used when $\underline{d} \in \operatorname{Pic}(C)$.

From (2.3) and (2.4), any element of $\operatorname{Pic}(F)$ corresponds to a divisor on $F$ of the form

$$
n H+\underline{d} f, n \in \mathbb{Z}, \underline{d} \in \operatorname{Pic}(C) .
$$

As an element of $\operatorname{Num}(F)$ this is

$$
n H+d f, n \in \mathbb{Z}, d=\operatorname{deg}(\underline{d}) \in \mathbb{Z} .
$$

Definition 2.5. For any $n \geq 0$ and for any $\underline{d} \in \operatorname{Div}(C)$, the linear system $|n H+\underline{d} f|$, if not empty, is said to be $n$-secant to the fibration $F \stackrel{\rho}{\rightarrow} C$ since its general element meets $f$ at $n$ points.

For any $\underline{d} \in \operatorname{Div}(C)$ such that $|H+\underline{d} f| \neq \emptyset$, any $B \in|H+\underline{d} f|$ is called a unisecant curve to the fibration $F \stackrel{\rho}{\rightarrow} C$ (or simply of $F$ ).

Any irreducible unisecant curve $B$ of $F$ is smooth and is called a section of $F$.

Recall that there is a one-to-one correspondence between sections $B$ of $F$ and surjections $\mathcal{F} \rightarrow L$, with $L=L_{B}$ a line bundle on $C$ (cf. [18, $\S \mathrm{V}$, Prop. 2.6 and 2.9]). Then, one has an exact sequence

where $N$ is a line bundle on $C$.

$$
0 \rightarrow N \rightarrow \mathcal{F} \rightarrow L \rightarrow 0
$$

Note that the surjection in (2.6) induces the inclusion $B=\mathbb{P}(L) \subset \mathbb{P}(\mathcal{F})=F$. If $L=$ $\mathcal{O}_{C}(\underline{m})$, with $\underline{m} \in \operatorname{Div}^{m}(C)$, then $m=H B$ and $B \sim H+(\underline{m}-\operatorname{det}(\mathcal{F})) f$.

For example, if $B \in|H|$, the associated exact sequence is

$$
0 \rightarrow \mathcal{O}_{C} \rightarrow \mathcal{F} \rightarrow \operatorname{det}(\mathcal{F}) \rightarrow 0,
$$

where the map $\mathcal{O}_{C} \hookrightarrow \mathcal{F}$ gives a global section of $\mathcal{F}$ which corresponds to the global section of $\mathcal{O}_{F}(1)$ vanishing on $B$.

With $B$ and $F$ as in Definition 2.5, from (2.6)

$$
\mathcal{O}_{B}(B) \cong N^{\vee} \otimes L
$$

(cf. [18, § 5]). In particular,

$$
B^{2}=\operatorname{deg}(L)-\operatorname{deg}(N)=d-2 \operatorname{deg}(N)=2 m-d
$$

Similar considerations hold if $B_{1}$ is a (reducible) unisecant curve of $F$ such that $H B_{1}=m$. Indeed, there exists a section $B \subset F$ and an effective divisor $\underline{a} \in \operatorname{Div}(C), a:=\operatorname{deg}(\underline{a})$, such that

$$
B_{1}=B+\underline{a} f
$$


where $B H=m-a$. In particular there exists a line bundle $L=L_{B}$ on $C$, with $\operatorname{deg}(L)=m-a$, fitting in a sequence like (2.6) . Consider the evaluation map

$$
e v: \mathcal{F} \rightarrow \mathcal{O}_{\underline{a}}^{\oplus 2} \text {. }
$$

In a local trivialization around the points in $\underline{a} \mathcal{F}$ splits as the sum of $N$ and $L$. Therefore, a local section $s$ of $\mathcal{F}$ around $\underline{a}$, can be considered as a pair $\left(s_{1}, s_{2}\right)$ where $s_{1}$ (respectively $s_{2}$ ) is a local section of $N$ (respectively of $L$ ). Then, $e v(s)=\left(e v\left(s_{1}\right), e v\left(s_{2}\right)\right)$, where we denoted by $e v$ also the obvious evaluation maps for $N$ and $L$. By projecting onto the diagonal of $\mathcal{O}_{\underline{a}}^{\oplus 2}$ we have a surjection

and therefore also a surjection of

$$
\mathcal{F} \rightarrow \mathcal{O}_{\underline{a}}
$$

$$
\mathcal{F} \rightarrow L \oplus \mathcal{O}_{\underline{a}}
$$

and it is clear now that it fits into the exact sequence

$$
0 \rightarrow N \otimes \mathcal{O}_{C}(-\underline{a}) \rightarrow \mathcal{F} \rightarrow L \oplus \mathcal{O}_{\underline{a}} \rightarrow 0 .
$$

As above, the surjection in (2.10) induces the inclusion $B_{1} \subset F$.

Definition 2.11. Let $S$ be a scroll of degree $d$ and genus $g$ as in Definition 2.2 corresponding to the pair $(\mathcal{F}, C)$. Let $B \subset F$ be a section and $L$ as in (2.6).

Let $\Gamma:=\Phi(B) \subset S$. If $\left.\Phi\right|_{B}$ is birational to the image, then $\Gamma$ is called a section of the scroll $S$ (the classical terminology for $\Gamma$ was directrix of $S$, cf. e.g. [9, Defn. 1.9]) .

We will say that the pair $(S, \Gamma)$ is associated with (2.6) and that $\Gamma$ corresponds to the line bundle $L$ on $C$.

If $m=\operatorname{deg}(L)$, then $\Gamma$ is a section of degree $m$ of $S$; moreover,

$$
\left.\Phi\right|_{B}: B \cong C \rightarrow \Gamma
$$

is determined by the linear series $\Lambda \subseteq|L|$, which is the image of the map

$$
H^{0}(\mathcal{F}) \rightarrow H^{0}(L) .
$$

More generally, if $B_{1} \subset F$ is a (reducible) unisecant curve and $\left.\Phi\right|_{B_{1}}$ is birational to the image, then we call $\Phi\left(B_{1}\right)=\Gamma_{1}$ a unisecant curve of degree $m$ of $S$. Note that such a curve is the union of a section and of some rulings.

As above, the pair $\left(S, \Gamma_{1}\right)$ corresponds to a sequence of the type (2.10).

In general, the map $\left.\Phi\right|_{B}$ may well be not an isomorphism, not even birational to the image (cf. Example 3.7); indeed, $\left.\Phi\right|_{B}$ can even contract $B$ to a point if $L \cong \mathcal{O}_{C}$, in which case $S$ is a cone (cf. Lemma 3.5).

When $g=0$ we have rational scrolls and these are well-known (see e.g. [15]). Thus, from now on, we shall focus on the case $g \geq 1$.

\section{Preliminary Results on SCROlls}

In this section, we collect some preliminary results concerning scrolls (cf. [29], [8] and [9]).

Let $C$ be a smooth, projective curve of genus $g$ and let $\mathcal{F}$ be a rank-two vector bundle on $C$ as in Assumptions 2.1.

If $K_{F}$ denotes a canonical divisor of $F$, one has that

$$
K_{F} \equiv-2 H+(d+2 g-2) f
$$


where $\equiv$ is the numerical equivalence on $\operatorname{Div}(F)$ (see e.g. [18]). From (3.1), Serre duality and Riemann-Roch theorem we have

$$
R+1:=h^{0}\left(\mathcal{O}_{F}(1)\right)=d-2 g+2+h^{1}\left(\mathcal{O}_{F}(1)\right) .
$$

Definition 3.2. The non-negative integer $h^{1}\left(\mathcal{O}_{F}(1)\right)$ is called the speciality of the scroll and will be denoted by $h^{1}(F)$, or simply by $h^{1}$, if there is no danger of confusion. Thus,

$$
R=d-2 g+1+h^{1}
$$

and the pair $(\mathcal{F}, C)$ determines $S \subset \mathbb{P}^{R}$ as a linearly normal scroll of degree $d$, genus $g$ and speciality $h^{1}$. Such a scroll $S$ is said to be special if $h^{1}>0$, non-special otherwise.

This definition coincides with the classical one given by Segre in [29, §3, p. 128]: Segre denotes by $n$ the degree of the scroll, by $p$ the sectional genus and by $i:=g-h^{1}$.

Since $R \geq 3$, then $d \geq 2 g+2-h^{1}$. In particular, for non-special scrolls

$$
d \geq 2 g+2 \text {. }
$$

The following lemma provides an upper-bound for the speciality (cf. [29, §14], [11] and [3, Lemma 5.7]).

Lemma 3.5. Let $C$ be a smooth, projective curve of genus $g \geq 1$ and let $F=\mathbb{P}(\mathcal{F})$ be a ruled surface on $C$ as in Assumption [2.1. If $\operatorname{det}(\mathcal{F})$ is non-special, then

$$
h^{1} \leq g \text {. }
$$

In addition, if $d \geq 2 g+2$, the equality holds in (3.6) if and only if $\mathcal{F}=\mathcal{O}_{C} \oplus L$, in which case $\Phi=\Phi_{\left|\mathcal{O}_{F}(1)\right|}$ maps $F$ to a cone $S$ over a projectively normal curve of degree $d$ and genus $g$ in $\mathbb{P}^{d-g}$.

Proof. The bound (3.6) follows from the exact sequence (2.7), corresponding to a smooth element $H \in\left|\mathcal{O}_{F}(1)\right|$ (cf. (2.8)) .

If the equality holds, then $h^{0}\left(\mathcal{O}_{F}(1)\right)=h^{0}(\mathcal{F})=d-g+2$. If $B \in|H|$ is the curve corresponding to the section of $\mathcal{F}$ given by $(2.7)$, then $\Phi(B)$ is a smooth curve of degree $d$ and genus $g$, which is linearly normal in $\mathbb{P}^{d-g}$. This curve is projectively normal (cf. [5], 23. and [24]). Therefore $F$ is mapped via $\Phi$ to a surface $S$ which is projectively normal, since its general hyperplane section is (cf. [14, Theorem 4.27]). In particular, $h^{1}\left(\mathcal{O}_{S}\right)=0$.

Since $S$ is birational to a ruled surface of positive genus, then $S$ cannot be smooth, and it has some normal (isolated) singularities. This forces $S$ to be a cone (cf. [7, Claim 4.4]). Hence, the assertion follows.

From (3.3) and from Lemma 3.5, we have

$$
d-2 g+1 \leq R \leq d-g+1
$$

where the upper-bound is realized by cones whereas the lower-bound by non-special scrolls (cf. [3. Theorem 5.4]). Any intermediate value of $h^{1}$ can be realized, e.g. by using decomposable vector bundles, as the following example shows (see [29, pp. 144-145] and [3. Example 5.11]).

Example 3.7. Let $g \geq 3, d \geq 4 g-1$ and $1 \leq h^{1} \leq g-1$ be integers. Let $L$ be a line bundle on $C$, such that $|L|$ is base-point-free and $h^{1}(L)=h^{1}$.

Let $D$ be a general divisor on $C$ of degree $d-\operatorname{deg}(L)$. Notice that, since $\operatorname{deg}(L) \leq 2 g-2$, then $\operatorname{deg}(D) \geq 2 g+1$, so the linear series $|D|$ is very ample.

Consider $\mathcal{F}=L \oplus \mathcal{O}_{C}(D)$. If $F=\mathbb{P}(\mathcal{F})$ then $\left|\mathcal{O}_{F}(1)\right|$ is base-point-free and $h^{1}(F)=h^{1}$.

For large values of $h^{1},\left|\mathcal{O}_{F}(1)\right|$ is rarely very ample (cf. the case $h^{1}=g$ in Lemma 3.5). For example 
(i) if $h^{1}=g-1$, then $|L|$ is a $g_{2}^{1}$ on $C$. In this case, $S$ has a double line $\Gamma$ because $\left|\mathcal{O}_{F}(1)\right|$ restricts as the $g_{2}^{1}$ to the section corresponding to the surjection $\mathcal{F} \rightarrow L$

(ii) if $h^{1}=g-2$, then either $C$ is hyperelliptic and $|L|=2 g_{2}^{1}$, or $C$ is trigonal and $|L|=g_{3}^{1}$ or $g=3$ and $L=\omega_{C}$. In the former case, as in $(i), S$ contains a double conic $\Gamma$; in the second case, $S$ has a triple line. Only in the latter case, $S$ may be smooth, and contains a smooth, plane quartic as a section.

The analysis of the interplay between the $h^{1}$ and the smoothness of the scroll is rather subtle in general, and we do not dwell on this here. For other examples, we refer the reader to [29, pp. 144-145], and to [2], [10] and [13].

We now want to discuss general properties of some unisecant curves on scrolls.

Definition 3.8. Let $\Gamma_{1} \subset S$ be a unisecant curve of $S$ of degree $m$ such that $\left(S, \Gamma_{1}\right)$ is associated to

$$
0 \rightarrow N \rightarrow \mathcal{F} \rightarrow L \oplus \mathcal{O}_{\underline{a}} \rightarrow 0
$$

where $\underline{a} \in \operatorname{Div}(C)$, possibly $\underline{a}=0$. Denote by $\Gamma$ the unique section contained in $\Gamma_{1}$. We will say that:

(i) $\Gamma_{1}$ is special if $h^{1}(C, L)>0$;

(ii) $\Gamma_{1}$ is linearly normally embedded if $H^{0}(\mathcal{F}) \rightarrow H^{0}\left(L \oplus \mathcal{O}_{\underline{a}}^{\oplus 2}\right)$.

If $\Gamma_{1}=\Gamma$ then $(i i)$ is equivalent to $\Gamma=\Phi_{|L|}(C)$.

By Definition 3.2 and Formulae (3.3), (3.4) and (3.9), we immediately have:

Proposition 3.10. Let $S \subset \mathbb{P}^{R}$ be a linearly normal scroll of degree $d \geq 2 g+2$, genus $g \geq 1$ and speciality $h^{1} \geq 0$, determined by the pair $(\mathcal{F}, C)$.

(i) Let $\Gamma_{1} \subset S$ be a unisecant curve of $S$, which is linearly normally embedded, and let $\left(S, \Gamma_{1}\right)$ be associated with (3.9). Then:

- if at least one of the two line bundles $L$ and $N$ in (3.9) is special, then $S$ is a special scroll;

- if both the line bundles $L$ and $N$ are non-special, then $S$ is non-special and

$$
R+1=h^{0}(\mathcal{F})=h^{0}(L)+h^{0}(N)+2 a .
$$

(ii) Conversely, the speciality of any unisecant curve of $S$ is less than or equal to the speciality of $S$. In particular, if $S$ is non-special, then $S$ contains only non-special unisecant curves.

Moreover, we have:

Proposition 3.11. Let $S \subset \mathbb{P}^{R}$ be a linearly normal, non-special scroll of genus $g \geq 1$ and degree $d \geq 2 g+2$. Then each unisecant curve of $S$ of degree

$$
m \leq d-2 g+1
$$

is linearly normally embedded in $S$.

Proof. Assume by contradiction that $\Gamma$ is not linearly normally embedded in $S$, i.e. the map $H^{0}(\mathcal{F}) \rightarrow H^{0}\left(L \oplus \mathcal{O}_{C}(\underline{a})\right)$ is not surjective. Equivalently, $h^{1}(C, N)>0$. Hence, $|N|=$ $g_{d-m}^{d-m-g+h^{1}(N)}$ is a special linear series on $C$.

From Clifford's Theorem

$$
2\left(d-m-g+h^{1}(N)\right) \leq d-m
$$

which gives

$$
m \geq d-2 g+2 h^{1}(N) \geq d-2 g+2
$$

which is contrary to the assumptions. 
The two results above are stated in [29, $\S 4$, p. 130 and p. 137] and proved apparently in a rather intricate way.

\section{RESUlts ON RANK-TWO VECTOR BUNDLES ON CURVES}

We recall here some results on rank-two vector bundles on curves which are frequently used in what follows. For complete details, we refer the reader to e.g. [26] and [31].

Let $C$ be a smooth, projective curve of genus $g \geq 0$. Let $\mathcal{E}$ be a vector bundle of rank $r \geq 1$ on $C$. The slope of $\mathcal{E}$, denoted by $\mu(\mathcal{E})$, is defined as

$$
\mu(\mathcal{E}):=\frac{\operatorname{deg}(\mathcal{E})}{r} \text {. }
$$

From now on, we shall be interested in the rank-two case.

A rank-two vector bundle $\mathcal{F}$ on $C$ is said to be indecomposable, if it cannot be expressed as a direct sum $L_{1} \oplus L_{2}$, for some $L_{i} \in \operatorname{Pic}(C), 1 \leq i \leq 2$, and decomposable otherwise.

Furthermore, $\mathcal{F}$ is said to be:

- semistable, if for any sub-line bundle $N \subset \mathcal{F}$, one has $\operatorname{deg}(N) \leq \mu(\mathcal{F})$;

- stable, if for any sub-line bundle $N \subset \mathcal{F}$, one has $\operatorname{deg}(N)<\mu(\mathcal{F})$;

- strictly semistable, if it is semistable and there is a sub-line bundle $N \subset \mathcal{F}$ such that $\operatorname{deg}(N)=\mu(\mathcal{F})$

- unstable, if there is a sub-line bundle $N \subset \mathcal{F}$ such that $\operatorname{deg}(N)>\mu(\mathcal{F})$. In this case, $N$ is called a destabilizing sub-line bundle of $\mathcal{F}$.

Recall the following well-known fact:

Lemma 4.2. In the above setting, if $\mathcal{F}=L_{1} \oplus L_{2}$ is decomposable, then it is unstable unless $\operatorname{deg}\left(L_{1}\right)=\operatorname{deg}\left(L_{2}\right)$, in which case $\mathcal{F}$ is strictly semistable.

Proof. Set $k=\operatorname{deg}\left(L_{1}\right)=\operatorname{deg}\left(L_{2}\right)$. Let $N \subset \mathcal{F}$ be any sub-line bundle; the injection $N \hookrightarrow \mathcal{F}$ gives rise to the injection

$$
\mathcal{O}_{C} \hookrightarrow \mathcal{F} \otimes N^{\vee}=\left(L_{1} \otimes N^{\vee}\right) \oplus\left(L_{2} \otimes N^{\vee}\right),
$$

i.e. to a global section of $\left(L_{1} \otimes N^{\vee}\right) \oplus\left(L_{2} \otimes N^{\vee}\right)$. Hence, there is $i \in\{1,2\}$ such that $L_{i} \otimes N^{\vee}$ is effective, i.e. $\operatorname{deg}(N) \leq \operatorname{deg}\left(L_{i}\right)=k=\mu(\mathcal{F})$, which shows the semistability of $\mathcal{F}$.

It is well-known that, given an integer $d$, there exists the moduli space of rank-two, semistable vector bundles of degree $d$ on $C$, which we denote by $U_{C}(d)$. This is a projective variety, and we denote by $U_{C}^{s}(d) \subseteq U_{C}(d)$ the open subscheme parametrizing stable bundles.

When $g=0$, every vector bundle of rank higher than one is decomposable (cf. e.g. [28, Thm. 2.1.1]). Furthermore, there is no stable vector bundle of rank $r>1$ on $\mathbb{P}^{1}$ (see e.g. [26, Corollary 5.2.1]). In particular, $U_{\mathbb{P} 1}^{s}(d)=\emptyset$ for any $d$.

When $g=1$, we have to distinguish two cases. If $d$ is odd, $U_{C}(d)=U_{C}^{s}(d) \cong C$ and every $[\mathcal{F}] \in U_{C}(d)$ is indecomposable. If $d$ is even, $U_{C}^{s}(d)=\emptyset$ (cf. [26, Rem. 5.9]), $U_{C}(d) \cong \operatorname{Sym}^{2}(C)$ and every $[\mathcal{F}] \in U_{C}(d)$ is the direct sum of two sub-line bundles, each of degree $d / 2$. For details, see [34, Theorem 16] and the proof of Theorem 5.4-(i) later on.

For $g \geq 2$, we have the following picture:

(i) $U_{C}(d)$ is irreducible, normal, of dimension $4 g-3$;

(ii) $U_{C}^{s}(d)$ coincides with the set of smooth points of $U_{C}(d)$.

More precisely (cf. [26, §5]):

(1) if $d$ is odd, then $U_{C}(d)=U_{C}^{s}(d)$ is smooth, i.e. each semistable vector bundle is stable; 
(2) if $d$ is even, there are strictly semistable vector bundles, i.e. the inclusion $U_{C}^{s}(d) \subset$ $U_{C}(d)$ is proper.

Proposition 4.3. Let $C$ be a smooth, projective curve of genus $g \geq 1$ and let $d$ be a positive integer.

(i) If $d \geq 4 g-3$ then, for any $[\mathcal{F}] \in U_{C}(d), h^{1}(C, \mathcal{F})=0$;

(ii) if $g \geq 2$ and $d \geq 2 g$ then, for $[\mathcal{F}] \in U_{C}(d)$ general, $h^{1}(C, \mathcal{F})=0$.

Proof. (i) For the proof see [26, Lemma 5.2].

(ii) We use a degeneration argument. Two cases must be considered.

(a) Assume $d=2 k$, with $k \geq g$. Let $L_{1}, L_{2} \in \operatorname{Pic}^{k}(C)$ be general line bundles. Let

$$
\mathcal{F}_{0}:=L_{1} \oplus L_{2} \text {. }
$$

Since $h^{1}\left(L_{i}\right)=0$, for $1 \leq i \leq 2$, then $h^{1}\left(\mathcal{F}_{0}\right)=0$. Observe that $\mu\left(\mathcal{F}_{0}\right)=k$. By Lemma 4.2, $\mathcal{F}_{0}$ is semistable, i.e. $\left[\mathcal{F}_{0}\right] \in U_{C}(2 k)$. By semicontinuity, $h^{1}(\mathcal{F})=0$ for general $[\mathcal{F}] \in U_{C}(2 k)$.

(b) Assume $d=2 k+1$, so $k \geq g$. Let $L \in \operatorname{Pic}^{k+1}(C)$ and $N \in \operatorname{Pic}^{k}(C)$ be general line bundles. Let $\mathcal{F}_{0}$ be a general rank-two vector bundle fitting in the exact sequence

$$
0 \rightarrow N \rightarrow \mathcal{F}_{0} \rightarrow L \rightarrow 0 \text {. }
$$

As before, since $h^{1}(L)=h^{1}(N)=0$, then $h^{1}\left(\mathcal{F}_{0}\right)=0$. Furthermore, $\mu\left(\mathcal{F}_{0}\right)=k+\frac{1}{2}$. We want to show that $\mathcal{F}_{0}$ is stable.

Since $\mathcal{F}_{0}$ corresponds to the general element in $\operatorname{Ext}^{1}(L, N)$, then the sequence (4.4) is unsplit, since $\operatorname{dim}\left(\operatorname{Ext}^{1}(L, N)\right)=h^{1}\left(N \otimes L^{\vee}\right)=g$.

Let $T \subset \mathcal{F}_{0}$ be any sub-line bundle. We have the following commutative diagram:

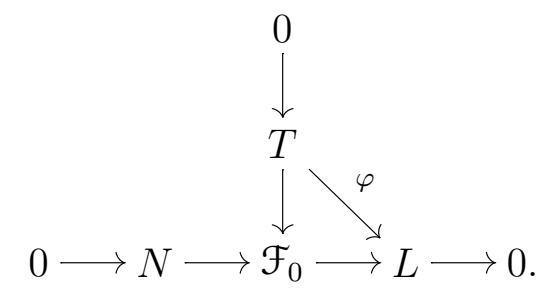

(i) If $\varphi$ is the zero-map, then $T$ is a sub-line bundle of $N$, so $\operatorname{deg}(T) \leq k<\mu\left(\mathcal{F}_{0}\right)$.

(ii) If $\varphi$ is not the zero-map, then $\varphi$ is injective, hence $L \otimes T^{\vee}$ is effective. Thus, $\operatorname{deg}(T) \leq k+1$ and the equality holds if and only if $L \cong T$. In the latter case, the exact sequence

$$
0 \rightarrow N \rightarrow \mathcal{F}_{0} \rightarrow L \rightarrow 0
$$

would split, against the assumption.

Therefore, $\operatorname{deg}(T)<\mu\left(\mathcal{F}_{0}\right)$, i.e. $\left[\mathcal{F}_{0}\right] \in U_{C}^{s}(2 k+1)$. One concludes the arguments using semicontinuity.

\section{HilBERT SCHEMES OF NON-SPECIAL SCROLLS}

From now on, we shall focus on linearly normal, non-special scrolls $S$ of degree $d$ and genus g. Therefore, from (3.3), $S \subset \mathbb{P}^{R}$ where

$$
R=d-2 g+1
$$

with $d$ as in (3.4).

If we moreover assume that $S$ is smooth, of genus $g \geq 1$, one can deduce further restrictions on $d$. Indeed, one has linearly normal, non-special smooth scrolls of genus $g \geq 1$ only for

$$
d \geq 5, \text { when } g=1, \text { and } d \geq 2 g+4, \text { when } g \geq 2,
$$


(cf. [3, Remark 4.20]).

Basic information about the Hilbert scheme of these scrolls are essentially contained in [2]. We recall the main results. First of all the following theorem (see [2], for a more explicit statement cf. Theorem 1.2 in [3]).

Theorem 5.3. Let $g \geq 0$ be an integer and set $k:=\min \{1, g-1\}$. If $d \geq 2 g+3+k$, then there exists a unique, irreducible component $\mathcal{H}_{d, g}$ of the Hilbert scheme of scrolls of degree $d$, sectional genus $g$ in $\mathbb{P}^{R}$ such that the general point $[S] \in \mathcal{H}_{d, g}$ represents a smooth, non-special and linearly normal scroll $S$.

Furthermore,

(i) $\mathcal{H}_{d, g}$ is generically reduced;

(ii) $\operatorname{dim}\left(\mathcal{H}_{d, g}\right)=7(g-1)+(d-2 g+2)^{2}=7(g-1)+(R+1)^{2}$;

(iii) $\mathcal{H}_{d, g}$ dominates the moduli space $\mathcal{M}_{g}$ of smooth curves of genus $g$.

For $g \geq 1$, the next result gives more information about the general scroll parametrized by $\mathcal{H}_{d, g}$ (cf. [2]; we give here a short, independent proof).

Theorem 5.4. Let $g \geq 1$ and let $d$ and $R$ be as in Theorem 5.3. Let $[S] \in \mathcal{H}_{d, g}$ be a general point and $(\mathcal{F}, C)$ be a pair which determines $S$, where $[C] \in \mathcal{M}_{g}$ general. Then $[\mathcal{F}]$ is a general point in $U_{C}(d)$.

Denote by $G_{S} \subset P G L(R+1, \mathbb{C})$ the subgroup of projective transformations fixing $S$.

(i) If $g=1$, then

- when $d$ is odd, then $G_{S}=\{1\}$ and the pair $(\mathcal{F}, C)$ determining $S$ is unique.

- when $d$ is even, then $\operatorname{dim}\left(G_{S}\right)=1$, and accordingly there is a 1-dimensional family of vector bundles $[\mathcal{F}] \in U_{c}(d)$, such that the pairs $(\mathcal{F}, C)$ determine $S$.

If $g \geq 2$, then $G_{S}=\{1\}$ and the pair $(\mathcal{F}, C)$ determining $S$ is unique.

Proof. We first consider the case $g \geq 2$. Denote by $\mathcal{U}_{d} \stackrel{\tau}{\rightarrow} \mathcal{M}_{g}$ the relative moduli stack of degree $d$, semistable, rank-two vector bundles so that, for $[C] \in \mathcal{M}_{g}, \tau^{-1}([C])=U_{C}(d)$. Since, for any $[C] \in \mathcal{M}_{g}, U_{C}(d)$ is irreducible of dimension $4 g-3$, then $\mathcal{U}_{d}$ is irreducible, of dimension $7 g-6$.

Let $\mathcal{F}_{\mathfrak{U}} \stackrel{\pi}{\longrightarrow} \mathcal{U}_{d}$ be the universal bundle. From Proposition 4.3 - (ii), on an open, dense subscheme $U \subseteq \mathcal{U}_{d},\left.\pi_{*}\left(\mathcal{F}_{\mathfrak{U}}\right)\right|_{U}$ is a vector bundle of rank $R+1$. After possibly shrinking $U$, we may assume that this vector bundle is trivial on $U$ and we can choose indipendent global sections $s_{0}, \ldots, s_{R}$ of $\left.\pi_{*}\left(\mathcal{F}_{\mathcal{U}}\right)\right|_{U}$.

Consider $\mathcal{P}_{\mathfrak{U}}:=U \times P G L(R+1, \mathbb{C})$ which is irreducible, of dimension $7 g-7+(R+1)^{2}$. An element of $\mathcal{P}_{\mathcal{U}}$ can be regarded as a triple $(C, \mathcal{F}, \rho)$, where $[C] \in \mathcal{M}_{g}$ is a general curve, $[\mathcal{F}] \in U_{C}(d)$ is general, and $\rho$ is a projective transformation. Moreover, the sections $s_{0}, \ldots, s_{R}$ induce indipendent sections of $H^{0}(C, \mathcal{F})$ and therefore determine a morphism $F=\mathbb{P}(\mathcal{F}) \rightarrow$ $S \subset \mathbb{P}^{R}$.

Let $\operatorname{Hilb}(d, g)$ denote the Hilbert scheme of scrolls of degree $d$ and genus $g$ in $\mathbb{P}^{R}$. Consider the morphism

$$
\Psi: \mathcal{P}_{\mathcal{U}} \rightarrow \operatorname{Hilb}(d, g)
$$

which maps the triple $(C, \mathcal{F}, \rho)$ to the surface $\rho(S)$.

Let $\mathcal{S}_{U}$ be the image of $\Psi$, which is irreducible. From Proposition 4.3 and Theorem 5.3 , $\mathcal{S}_{U}$ is contained in $\mathcal{H}_{d, g}$. Since semistability is an open condition (cf. e.g. [19, Proposition 2.3.1]), then $\Psi$ is dominant onto $\mathcal{H}_{d, g}$.

Recalling the description of $U_{C}(d)$ in $\S$, it follows that the general scroll parametrized by a point in $\mathcal{H}_{d, g}$ corresponds to a stable vector bundle. 
From Theorem 5.3, we deduce that $\Psi$ is generically finite onto the image. More precisely, let $(C, \mathcal{F}, \rho)$ and $\left(C, \mathcal{F}^{\prime}, \rho^{\prime}\right)$ be points in $\mathcal{P}_{u}$ such that $\rho(S)=\Psi(C, \mathcal{F}, \rho)=\Psi\left(C, \mathcal{F}^{\prime}, \rho^{\prime}\right)=\rho\left(S^{\prime}\right)$. Hence $\phi=\left(\rho^{\prime}\right)^{-1} \circ \rho: S \rightarrow S^{\prime}$ is a projective transformation. Let $F=\mathbb{P}(\mathcal{F}) \cong \mathbb{P}\left(\mathcal{F}^{\prime}\right)=F^{\prime}$. Since $C$, being general, has no non-trivial automorphisms, $\phi$ induces an automorphism $\Phi$ : $F \rightarrow F^{\prime}$ and $\Phi^{*}\left(\mathcal{O}_{F^{\prime}}(1)\right)=\mathcal{O}_{F}(1)$.

Since $\mathbb{P}(\mathcal{F}) \cong \mathbb{P}\left(\mathcal{F}^{\prime}\right)$, there is a line bundle $\eta \in \operatorname{Pic}^{0}(C)$ such that $\mathcal{F}^{\prime} \cong \mathcal{F} \otimes \eta$. Note that $\operatorname{Pic}^{0}(C) \subset \operatorname{Pic}^{0}(F)=\operatorname{Pic}^{0}\left(F^{\prime}\right)$. Thus, $\mathcal{O}_{F}(1) \cong \Phi^{*}\left(\mathcal{O}_{F^{\prime}}(1)\right) \otimes \eta$. This implies that $\eta$ is trivial.

Finally, we claim that $\rho=\rho^{\prime}$, i.e. $\phi=$ id. Otherwise, $\Phi: F \rightarrow F$ would come from a non-trivial automorphism of $\mathcal{F}$ which is not possible because $\mathcal{F}$, being stable, is simple (cf. e.g. [26, Corollary 5.1.1]).

Now let $g=1$. As above, we can consider $\mathcal{U}_{d} \stackrel{\tau}{\rightarrow} \mathcal{M}_{1}$, the open subset $U \subset \mathcal{U}_{d}$ and the variety $\mathcal{P}_{\mathfrak{U}}=U \times P G L(R+1, \mathbb{C})$. Then, $\mathcal{P}_{\mathfrak{U}}$ is irreducible and

$$
\operatorname{dim}\left(\mathcal{P}_{\mathfrak{u}}\right)= \begin{cases}d^{2}+1, & \text { if } d \text { is odd } \\ d^{2}+2, & \text { if } d \text { is even, }\end{cases}
$$

(cf. §(4). For the same reasons as above, the map $\Psi: \mathcal{P}_{\mathfrak{U}} \rightarrow \mathcal{H}_{d, 1}$ is dominant.

If $[S] \in \mathcal{H}_{d, 1}$ denotes the general point and if $\mathcal{P}_{S}:=\Psi^{-1}([S])$, we have:

$$
\operatorname{dim}\left(\mathcal{P}_{S}\right)= \begin{cases}1, & \text { if } d \text { is odd } \\ 2, & \text { if } d \text { is even }\end{cases}
$$

Indeed, assume that $S$ is determined by a pair $(\mathcal{F}, C)$. Let $h=$ g.c.d. $(2, d), 2=h n^{\prime}$ and $d=h d^{\prime}$. Then, as in [34, Theorem 16], any [F] $\in U_{C}(d)$ is of the form

$$
\mathcal{F}=E\left(n^{\prime}, d^{\prime}\right) \otimes\left(\bigoplus_{i=1}^{h} L_{i}\right),
$$

where $E\left(n^{\prime}, d^{\prime}\right)$ is the Atiyah's bundle of rank $n^{\prime}$ and degree $d^{\prime}$ and where the $L_{i}$ 's are line bundles of degree 0 , determined up to multiplication by a $n^{\prime}$-torsion element in $J(C) \cong C$, $1 \leq i \leq h$.

If $d$ is odd, then $h=1, n^{\prime}=2, d=d^{\prime}$, so any [F] $\in U_{C}(d)$ is of the form $\mathcal{F}=E(2, d) \otimes L$, for some $L \in J(C)$. Since $C$ is an elliptic curve, for any $x \in C$ :

$$
\begin{aligned}
t_{x}: C & \cong C \\
p & \longrightarrow p+x,
\end{aligned}
$$

is an automorphism of $C$. Since $U_{C}(d) \cong C$, this defines a natural action of $C$ on $U_{C}(d)$ : fix $[\mathcal{F}] \in U_{C}(d)$, then for every $M \in J(C)$,

$$
t_{\left[M^{\vee}\right]}^{*}(\mathcal{F})=\mathcal{F} \otimes M \in U_{C}(d) .
$$

From [20], $t_{\left[M^{\vee}\right]} \in \operatorname{Aut}(C)$ lifts to an automorphism of the elliptic ruled surface $\mathbb{P}(\mathcal{F})$. As a consequence, for any other $\left[\mathcal{F}^{\prime}\right] \in U_{C}(d), \mathbb{P}(\mathcal{F}) \cong \mathbb{P}\left(\mathcal{F}^{\prime}\right)$. Since $\operatorname{dim}\left(\mathcal{P}_{S}\right)=1$, an argument completely similar to the one we made in the case $g \geq 2$ proves that $G_{S}=\{1\}$.

If $d$ is even, then $h=2, n^{\prime}=1$ and $d^{\prime}=d / 2$, so any [F] $\in U_{C}(d)$ is of the form $\mathcal{F}=$ $E(1, d / 2) \otimes\left(L_{1} \oplus L_{2}\right)$, for some $L_{1}, L_{2} \in J(C)$. In the same way, any other $\left[\mathcal{F}^{\prime}\right] \in U_{C}(d)$ of the form $\mathcal{F}^{\prime}=E(1, d / 2) \otimes\left(\left(L_{1} \otimes N\right) \oplus\left(L_{2} \otimes N\right)\right)$, for some $N \in J(C)$, and $\mathbb{P}(\mathcal{F}) \cong \mathbb{P}\left(\mathcal{F}^{\prime}\right)$. Since $\operatorname{dim}\left(\mathcal{P}_{S}\right)=2$, this implies that $\operatorname{dim}\left(G_{S}\right)=1$.

Remark 5.5. If $g=1$ and $d$ is even, the elements of the one-dimensional group $G_{S}$ correspond to non-trivial endomorphisms of the corresponding strictly semistable vector bundle $\mathcal{F}$. This can be read off by looking at the projective geometry of $S$. Indeed, $S$ contains sections $\Gamma_{i}$ 
of degree $d / 2$ associated with the line bundles $M_{i}=E(1, d / 2) \otimes L_{i}, 1 \leq i \leq 2$. These two sections are disjoint, since $\mathcal{F}$ is decomposable (cf. [18, p. 383]), and are the curves of minimal degree of $S$, since they are determined by quotients via sub-line bundles of maximal degree (cf. also Theorem 7.1).

Let $\Lambda_{i}:=\left\langle\Gamma_{i}\right\rangle \cong \mathbb{P}^{\frac{d-2}{2}}$, for $1 \leq i \leq 2$. Then $\Lambda_{1} \cap \Lambda_{2}=\emptyset$ so, if $G$ denotes the connected subgroup of $P G L(R+1, \mathbb{C})$ of elements which pointwise fix these two skew linear subspaces of $\mathbb{P}^{R}$, then $\operatorname{dim}(G)=1$ and each element of $G$ fixes $S$. This shows that $G$ is the connected component of the identity of $G_{S}$.

Remark 5.6. In [3], we gave an explicit dimension count for $\mathcal{H}_{d, g}$ (cf. [3, Theorem 5.4 and Remark 5.6]). Theorem 5.4 gives another way of making the same computation.

Precisely, when $g \geq 2$, the number of parameters on which the general point of $\mathcal{H}_{d, g}$ depends, is given by the following count:

- $3 g-3$ parameters for the class of the curve $C$ in $\mathcal{M}_{g}$, plus

- $4 g-3$ parameters for the general rank-two vector bundle in $U_{C}(d)$, plus

- $(R+1)^{2}-1$ parameters for projective transformations in $\mathbb{P}^{R}$.

When $g=1$, we have:

- 1 parameter for $C$ in $\mathcal{M}_{1}$, plus

- 1 or 2 parameters (according to the cases $d$ odd or $d$ even), for the general rank-two vector bundle in $U_{C}(d)$, plus

- $(R+1)^{2}-1-\operatorname{dim}\left(G_{S}\right)$ parameters for projective transformations in $\mathbb{P}^{R}$ (with $\operatorname{dim}\left(G_{S}\right)=$ 0 or 1 , according to the cases $d$ odd or $d$ even), minus

- 1 parameter, for the $C$-action on $U_{C}(d)$.

Remark 5.7. Even if $d$ is large with respect to $g$ (cf. Proposition 4.3 - (i)), Theorem 5.4 does not imply that all smooth scrolls in $\mathcal{H}_{d, g}$ come from either a stable or a semistable rank-two vector bundle on $C$. Indeed, let $\mathcal{F}$ be any rank-two vector bundle on a curve $C$ of genus $g$. By twisting $\mathcal{F}$ with a sufficiently high multiple of an ample line bundle $A$ on $C$, we have a new vector bundle $\mathcal{F}^{\prime}=\mathcal{F} \otimes A^{\otimes k}$, such that $h^{1}\left(C, \mathcal{F}^{\prime}\right)=0$ and $\mathcal{O}_{\mathbb{P}\left(\mathcal{F}^{\prime}\right)}(1)$ is very ample. By embedding $\mathbb{P}\left(\mathcal{F}^{\prime}\right)$ via $\left|\mathcal{O}_{\mathbb{P}\left(\mathcal{F}^{\prime}\right)}(1)\right|$, one has a smooth, non-special, linearly normal scroll $S$ of a certain degree $d$, and therefore $[S] \in \mathcal{H}_{d, g}$.

More precisely, look at the following example. Let $C$ be any smooth, projective curve of genus $g \geq 2$ and $k \geq 2$ be an integer. Let $L \in \operatorname{Pic}^{2 g+k}(C)$ and $N \in \operatorname{Pic}^{2 g+k-1}(C)$ be general line bundles. Let $\mathcal{F}_{0}$ be a general, rank-two vector bundle on $C$ fitting in the exact sequence

$$
0 \rightarrow L \rightarrow \mathcal{F}_{0} \rightarrow N \rightarrow 0 \text {. }
$$

Thus, $\operatorname{deg}\left(\mathcal{F}_{0}\right)=4 g+2 k-1$ and, by the generality assumption on $L$ and $N, h^{1}\left(C, \mathcal{F}_{0}\right)=0$. Furthermore, by degree assumptions, both $L$ and $N$ are very ample on $C$. Therefore, the pair $\left(\mathcal{F}_{0}, C\right)$ determines a smooth scroll $S$ which is non-special and linearly normal in $\mathbb{P}^{2 g+2 k}$, i.e. $[S] \in \mathcal{H}_{4 g+2 k-1, g}$.

Nonetheless, $\mathcal{F}_{0}$ is unstable on $C$ : indeed, $\mu\left(\mathcal{F}_{0}\right)=2 g+k-\frac{1}{2}$ whereas $\operatorname{deg}(L)=2 g+k>$ $\mu\left(\mathcal{F}_{0}\right)$, so $L$ is a destabilizing sub-line bundle of $\mathcal{F}_{0}$.

In accordance with Theorem 5.4, the reader can verify that the number of parameters on which scrolls of this type depend is at most

$$
6 g-5+(2 g+2 k+1)^{2}<\operatorname{dim}\left(\mathcal{H}_{4 g+2 k-1, g}\right) .
$$

Remark 5.8. In [3, Theorem 1.2] we proved that there are points in $\mathcal{H}_{d, g}$ corresponding to unions of planes with Zappatic singularities. This means that smooth surfaces in $\mathcal{H}_{d, g}$ degenerate to these unions of planes. It is interesting to remark that this applies to surfaces 
of the type $C \times \mathbb{P}^{1}$, suitably embedded as scrolls corresponding to points in $\mathcal{H}_{d, g}$. First of all, let $C$ be any curve of genus $g$ and let $L$ be a very-ample non-special line bundle of degree $d \geq g+3$. The global sections of $L$ determine an embedding of $C$ in $\mathbb{P}^{d-g}$. Consider the Segre embedding of $C \times \mathbb{P}^{1}$. This gives a linearly normal, non-special smooth scroll of degree $2 d$ in $\mathbb{P}^{2 d-2 g+1}$ and the corresponding point sits in $\mathcal{H}_{2 d, g}$. Moreover, by looking at the argument in [3, §3], one sees that the planar Zappatic surface $X_{2 d, g}$ contained in $\mathcal{H}_{2 d, g}$ is a limit of a product. In Zappa's original paper [35], the author remarked that products $C \times \mathbb{P}^{1}$ can be degenerated to union of quadrics, leaving as an open problem to prove the degeneration to union of planes.

We finish this section by constructing suitable reducible surfaces, frequently used in the rest of the paper, corresponding to points in $\mathcal{H}_{d, g}$. The first construction is contained in [3, Constructions 4.1, 4.2, Claim 4.3, Theorem 4.6, Lemma 4.7] (cf. also [6]), and will be stated below for the reader's convenience.

Construction 5.9. Let $g \geq 1$, $d$ and $\mathcal{H}_{d, g}$ be as in Theorem 5.3. Then $\mathcal{H}_{d, g}$ contains points $[T]$ such that $T$ is a reduced, connected, reducible surface, with global normal crossings, of the form

$$
T:=X \cup Q,
$$

where $X$ is a scroll corresponding to a general point of $\mathcal{H}_{d-2, g-1}$ and $Q$ is a smooth quadric, such that $X \cap Q=l_{1} \cup l_{2}$, where $l_{i}$ are general rulings of $X$, for $1 \leq i \leq 2$, and the intersection is transverse.

Furthermore, if $\mathcal{N}_{T / \mathbb{P}^{R}}$ denotes the normal sheaf of $T$ in $\mathbb{P}^{R}$, then $h^{1}\left(T, \mathcal{N}_{T / \mathbb{P}^{R}}\right)=0$; in particular, $[T]$ is a smooth point of $\mathcal{H}_{d, g}$.

The second construction is similar. Precisely, we have:

Construction 5.11. Let $g \geq 1$, $d$ and $\mathcal{H}_{d, g}$ be as in Theorem5.3. Then $\mathcal{H}_{d, g}$ contains points $[Y]$ such that $Y$ is a reduced, connected, reducible surface, with global normal crossings, of the form

$$
Y:=W \cup Q_{1} \cup \cdots \cup Q_{g},
$$

where $W$ is a rational normal scroll, corresponding to a general point of $\mathcal{H}_{d-2 g, 0}$, and each $Q_{j}$ is a smooth quadric, such that

$$
Q_{j} \cap Q_{k}=\emptyset, \text { if } 1 \leq j \neq k \leq g
$$

and

$$
W \cap Q_{j}=l_{1, j} \cup l_{2, j},
$$

where $l_{i, j}$ are general rulings of $W$, for $1 \leq i \leq 2,1 \leq j \leq g$, and the intersection is transverse.

Furthermore, for any such $Y$, one has $h^{1}\left(Y, \mathcal{N}_{Y / \mathbb{P}^{R}}\right)=0$; in particular, $[Y]$ is a smooth point of $\mathcal{H}_{d, g}$.

Proof. Let $[W] \in \mathcal{H}_{d-2 g, 0}$ be a general point. This corresponds to a smooth, rational normal scroll of degree $d-2 g$ in $\mathbb{P}^{R}$. Let $l_{1, j}, l_{2, j}, 1 \leq j \leq g$, be $2 g$ general rulings of $W$. Let $\Pi_{j}$ be the $\mathbb{P}^{3}$ spanned by $l_{1, j}$ and $l_{2, j}$. Let $Q_{j} \subset \Pi_{j}$ be a general quadric, containing $l_{1, j}, l_{2, j}$, for $1 \leq j \leq g$. Then, $Q_{j}$ is smooth and, for any $1 \leq j \leq g$,

$$
W \cap Q_{j}=l_{1, j} \cup l_{2, j}
$$

and the intersection is transverse (for $g=1$, we have only one quadric and the assertion follows, whereas for $g \geq 2$, see [3, Construction 4.2]). 
By generality and since $d \geq 2 g+4$, when $g \geq 2$, one sees that $\operatorname{dim}\left(\Pi_{j} \cap \Pi_{k}\right) \leq 1$, for $1 \leq j \neq k \leq g$, and therefore we can assume (5.13).

Let $Y$ be as in (5.12). Then $Y$ is of degree $d$, its sectional (arithmetic) genus is $g$ and $h^{1}\left(Y, \mathcal{O}_{Y}(1)\right)=0$ since it is clearly linearly normal in $\mathbb{P}^{R}$. From Theorem 5.3 , it follows that $[Y] \in \mathcal{H}_{d, g}$. Furthermore, as in the proof of [3, Theorem 4.6 and Lemma 4.7], $h^{1}\left(\mathcal{N}_{Y / \mathbb{P} R}\right)=0$ so $[Y]$ is a smooth point of $\mathcal{H}_{d, g}$. Thus, $[Y]$ deforms to a general point $[S] \in \mathcal{H}_{d, g}$.

\section{Properties of the sCHeme of unisecant CURVES}

In this section we prove Theorem 6.9 below, which contains basic information on the scheme parametrizing unisecant curves of given degree $m$ on the scroll $S$, where $[S]$ is a general point in $\mathcal{H}_{d, g}$, with $g \geq 0$ and $d$ as in Theorem 5.3.

First, we recall some results in [11, which are inspired by the work of C. Segre [29, $\S 11$, p. 138].

Definition 6.1 (see [11, Definition 6.1]). Let $C$ be a smooth, projective curve of genus $g \geq 0$. Let $F=\mathbb{P}(\mathcal{F})$ be a geometrically ruled surface over $C$ and let $d=\operatorname{deg}(\mathcal{F})$. For any positive integer $m$, denote by

$$
\operatorname{Div}_{F}^{1, m}
$$

the Hilbert scheme of unisecant curves of $F$, which are of degree $m$ with respect to $\mathcal{O}_{F}(1)$ (cf. Definition 2.5).

Remark 6.3. By recalling (2.6) and (2.10), the elements in $\operatorname{Div}_{F}^{1, m}$ correspond to quotients of $\mathcal{F}$. Therefore, $\operatorname{Div}_{F}^{1, m}$ has a natural structure as a Quot-scheme (cf. [17]). As such, Div ${ }_{F}^{1, m}$ has an expected dimension

$$
d_{m}:=\max \{-1,2 m-d-g+1\}
$$

and therefore

$$
\operatorname{dim}\left(\operatorname{Div}_{F}^{1, m}\right) \geq d_{m}
$$

Definition 6.6 (see [11, Definition 6.1]). Notation as in Definition 6.1, $F$ is said to be a general ruled surface if:

(i) $\operatorname{dim}\left(\operatorname{Div}_{F}^{1, m}\right)=d_{m}$;

(ii) $\operatorname{Div}_{F}^{1, m}$ is smooth, for any $m$ such that $d_{m} \geq 0$;

(iv) $\operatorname{Div}_{F}^{1, m}$ is irreducible, for any $m$ such that $d_{m}>0$.

Note that being general is an open condition in $\mathcal{H}_{d, g}$.

In [11, there is an asymptotic existence result for general ruled surfaces.

Theorem 6.7 (cf. [11, Théorème 7.1]). Let $C$ be a smooth, projective curve of genus $g \geq 0$. There is a positive integer $\delta_{C}$ such that, for every $d \geq \delta_{C}$, there is a general ruled surface of degree $d$ over $C$.

As a consequence, using [3, Proposition 5.2 and Theorem 5.4] and the proof of [11, Théorème 7.1], one has:

Corollary 6.8. For every $g \geq 0$, there is a positive integer $\delta_{g}$ such that, for any $d \geq \delta_{g}$, the general point of $\mathcal{H}_{d, g}$ corresponds to a general ruled surface.

Note that Ghione's argument gives no information about $\delta_{g}$. Using a degeneration argument, it is possible to improve Corollary 6.8 by specifing a bound on $\delta_{g}$. 
Theorem 6.9. Let $g$ and $d$ be as in Theorem 5.3. If $[S] \in \mathcal{H}_{d, g}$ is a general point, then $S$ is a general ruled surface.

Proof. We proceed by induction on $g$. The case $g=0$ is clear.

Assume $g>0$ and let either $d \geq 5$, if $g=1$, or $d \geq 2 g+4$, if $g \geq 2$. Let $[X] \in \mathcal{H}_{d-2, g-1}$ be a general point. By induction, $X$ is a smooth, general ruled surface. Let $l_{1}$ and $l_{2}$ be two general rulings of $X$. Let $Q$ be a general quadric surface containing $l_{1}$ and $l_{2}$; thus $Q$ is smooth and $X$ and $Q$ meet transversally along $X \cap Q=l_{1} \cup l_{2}$.

In particular, the surface $T:=X \cup Q$ is such as in Construction [5.9, so $[T]$ is a smooth point of $\mathcal{H}_{d, g}$.

We consider a section $\Gamma_{T}$ of $T$ as a connected union

$$
\Gamma_{T}=\Gamma_{X} \cup \Gamma_{Q}
$$

where $\Gamma_{X}$ (resp. $\Gamma_{Q}$ ) is a section of $X$ (resp. of $Q$ ), such that $\Gamma_{X}$ and $\Gamma_{Q}$ meet transversally at $\Gamma_{X} \cap \Gamma_{Q}=\left\{p_{1}, p_{2}\right\}$, where $p_{i} \in l_{i}, 1 \leq i \leq 2$. From Definition 2.11, since $X$ and $Q$ are smooth then $\Gamma_{X}$ and $\Gamma_{Q}$ are both smooth and irreducible, hence $\Gamma_{T}$ is a reduced, reducible curve having two nodes as its only singularities.

Similarly as in Definition 6.6, we will denote by

$$
\operatorname{Div}_{T}^{1, m}
$$

the Hilbert scheme of curves on $T$ of degree $m$, arithmetic genus $g$, which intersect at only one point the general line of the ruling of $X$ and of the ruling $\left|l_{1}\right|=\left|l_{2}\right|$ of $Q$.

Thus, $\left[\Gamma_{T}\right] \in \operatorname{Div}_{T}^{1, m}$ is the union of two curves

(i) $\left[\Gamma_{X}\right] \in \operatorname{Div}_{X}^{1, m_{X}}$, with $m_{X}<m$, and

(ii) $\left[\Gamma_{Q}\right] \in \operatorname{Div}_{Q}^{1, m-m_{X}}$,

which match along $l_{1} \cup l_{2}$.

By induction and by (6.4), we have:

- if $d+g-3$ is even, then $\frac{d+g-3}{2} \leq m_{X} \leq m-1$,

- if $d+g-3$ is odd, then $\frac{d+g-4}{2} \leq m_{X} \leq m-1$,

- in any case, by induction, $d_{m_{X}}=2 m_{X}-d-g+4=\operatorname{dim}\left(\operatorname{Div}_{X}^{1, m_{X}}\right)$.

Moreover, the scheme $\operatorname{Div}_{X}^{1, m_{X}}$ is smooth and, in addition, it is irreducible unless $d_{m_{X}}=0$. This is equivalent $d+g-3$ odd and $m_{X}=\frac{d+g-4}{2}$, in which case $\operatorname{Div}_{X}^{1, m_{X}}$ consists of finitely many distinct curves on $X$ (in Theorem 7.1 - (ii), we shall prove that the number of these curves is $\left.2^{g-1}\right)$. The scheme $\operatorname{Div}_{Q}^{1, m-m_{X}}$ is not empty, irreducible and $\operatorname{dim}\left(\operatorname{Div}_{Q}^{1, m-m_{X}}\right)=$ $2\left(m-m_{X}\right)-1 \geq 1$.

For any $m_{X}$ as above, let $G_{1}^{m_{X}}=\operatorname{Div}_{X}^{1, m_{X}}$ and $G_{2}^{m_{X}}=\operatorname{Div}_{Q}^{1, m-m_{X}}$. We have two natural rational maps

$$
\phi_{i}^{m_{X}}: G_{i}^{m_{X}} \rightarrow \mathbb{P}^{1} \times \mathbb{P}^{1}, \quad 1 \leq i \leq 2,
$$

given by intersecting the general curve in $G_{i}^{m_{X}}$ with $l_{1}$ and $l_{2}$. Consider

$$
G_{T}^{m_{X}}
$$

the closure of the fibre product of the maps $\phi_{i}^{m_{X}}$, for $1 \leq i \leq 2$.

Claim 6.13. Every irreducible component of $G_{T}^{m_{X}}$ has dimension $d_{m}=2 m-d-g+1 \geq 0$. Moreover, if $d_{m}>0$, then $G_{T}^{m_{X}}$ is irreducible. 
Proof of Claim 6.13. For the first part, observe that the family $G_{2}^{m_{X}}$ is the linear system of curves of type $\left(1, m-m_{X}-1\right)$ on $Q$ and $\operatorname{dim}\left(G_{2}^{m_{X}}\right)=2\left(m-m_{X}\right)-1$.

Consider the projection

$$
G_{T}^{m_{X}} \stackrel{\pi_{1}}{\longrightarrow} G_{1}^{m_{X}}
$$

(a) If $m-m_{X}>1$, then $\pi_{1}$ is surjective and its fibres are projective spaces of dimension $2\left(m-m_{X}\right)-3$; hence the assertion follows.

(b) If $m-m_{X}=1$, then $G_{T}^{m_{X}}$ is isomorphic to $\left(\phi_{1}^{m_{X}}\right)^{-1}\left(\phi_{2}^{m_{X}}\left(G_{2}^{m_{X}}\right)\right)$. Note that $\phi_{2}^{m_{X}}\left(G_{2}^{m_{X}}\right)$ is a smooth, rational curve of type $(1,1)$ on $\mathbb{P}^{1} \times \mathbb{P}^{1}$. Since $Q$ is general, this curve is general in its linear system. This implies that $\operatorname{dim}\left(G_{T}^{m_{X}}\right)=\operatorname{dim}\left(G_{1}^{m_{X}}\right)-1$, proving the first assertion.

The assertion about the irreducibility of $G_{T}^{m_{X}}$, when $d_{m}>0$, is clear in case (a) above. In case (b), by induction, $G_{1}^{m_{X}}$ is smooth and irreducible, since $d_{m_{X}}=d_{m}+1>0$. The proof of case (b) shows that $G_{T}^{m_{X}}$ is the pull-back via $\phi_{1}^{m_{X}}$ of a general curve of type $(1,1)$ on $\mathbb{P}^{1} \times \mathbb{P}^{1}$. This is irreducible by Bertini's theorem.

The general element of a component of $G_{T}^{m_{X}}$ is a pair $\left(\Gamma_{X}, \Gamma_{Q}\right)$ such that neither $\Gamma_{X}$ nor $\Gamma_{Q}$ contains either $l_{1}$ or $l_{2}$.

Let

$$
\mathcal{U}:=\bigcup_{\left\lfloor\frac{d+g-3}{2}\right\rfloor \leq j \leq m-1} G_{T}^{j},
$$

with $G_{T}^{j}$ as in (6.12). When $d_{m}=0$, then $\mathcal{U}=G_{T}^{\frac{d+g-3}{2}}$; when $d_{m}>0$, the irreducible components of $\mathcal{U}$ coincide with the $G_{T}^{j}$ 's (cf. Claim 6.13).

Note that there is a natural map

$$
\mathcal{U} \stackrel{\psi}{\longrightarrow} \operatorname{Div}_{T}^{1, m}
$$

which is surjective and birational on any irreducible component of $\mathcal{U}$. Therefore, the irreducible components of $\operatorname{Div}_{T}^{1, m}$ are images of the irreducible components of $\mathcal{U}$. In particular, by Claim 6.13,

$$
\operatorname{dim}\left(\operatorname{Div}_{T}^{1, m}\right)=d_{m}
$$

Claim 6.15. If $[S] \in \mathcal{H}_{d, g}$ is a general point and if $\operatorname{Div}_{S}^{1, m} \neq \emptyset$, then $d_{m} \geq 0$, i.e. $2 m-d-$ $g+1 \geq 0$, and moreover $\operatorname{dim}\left(\operatorname{Div}_{S}^{1, m}\right)=d_{m}$.

Proof of Claim 6.15. Note that, when $S$ degenerates to $T$, the flat limit of $\operatorname{Div}_{S}^{1, m}$ is contained in $\operatorname{Div}_{T}^{1, m}$. If $\operatorname{Div}_{S}^{1, m} \neq \emptyset$, then $\operatorname{Div}_{T}^{1, m} \neq \emptyset$. Therefore, there is a $\left\lfloor\frac{d+g-3}{2}\right\rfloor \leq j \leq m-1$ such $G_{T}^{j} \neq \emptyset$. Then $d_{m} \geq 0$ follows from Claim 6.13. By semicontinuity, one has

$$
\operatorname{dim}\left(\operatorname{Div}_{S}^{1, m}\right) \leq \operatorname{dim}\left(\operatorname{Div}_{T}^{1, m}\right)=d_{m}
$$

Equality holds by (6.5) .

The next step is the following:

Claim 6.16. If $d_{m}>0, \operatorname{Div}_{T}^{1, m}$ is connected.

Proof of Claim 6.16. Let $\operatorname{Div}_{T}^{1, m}(j)$ be the irreducible component of $\operatorname{Div}_{T}^{1, m}$ which is the image of $G_{T}^{j}$ via $\psi$. We will prove that, for every $j$ such that $\left\lfloor\frac{d+g-3}{2}\right\rfloor \leq j \leq m-2$, the subschemes $\operatorname{Div}_{T}^{1, m}(j)$ and $\operatorname{Div}_{T}^{1, m}(j+1)$ intersect.

Consider a general point of $\operatorname{Div}_{T}^{1, m}(j)$. This consists of the union of two general curves $\left[\Gamma_{1}\right] \in \operatorname{Div}_{X}^{1, j},\left[\Gamma_{2}\right] \in \operatorname{Div}_{Q}^{1, m-j}$ with $\Gamma_{1} \cap \Gamma_{2}=\left\{p_{1}, p_{2}\right\}$, where $p_{i} \in l_{i}, 1 \leq i \leq 2$. 
Since $m-j \geq 2$, the linear system $\left|\Gamma_{2}\right|$ has dimension at least three. Hence,

$$
\operatorname{dim}\left(\left|\mathcal{J}_{\left\{p_{1}, p_{2}\right\} / Q} \otimes \mathcal{O}_{Q}\left(\Gamma_{2}\right)\right|\right) \geq 1
$$

and therefore $\Gamma_{2}$ degenerates inside the latter linear system to $\bar{\Gamma}_{2}+l_{1}$, where $\bar{\Gamma}_{2} \in \operatorname{Div}_{Q}^{1, m-j-1}$ is a general curve on $Q$ passing through $p_{2}$ and intersecting $l_{1}$ at a point $q_{1}$.

Now, the curve $\left[\Gamma_{1}+l_{1}\right] \in \operatorname{Div}_{X}^{1, j+1}$. Since, by induction and by (6.4),

$$
\operatorname{dim}\left(\operatorname{Div}_{X}^{1, j+1}\right)=\operatorname{dim}\left(\operatorname{Div}_{X}^{1, j}\right)+2
$$

we can find a one-dimensional family of curves in $\operatorname{Div}_{X}^{1, j+1}$ passing through $q_{1}$ and $p_{2}$, and degenerating to $\Gamma_{1}+l_{1}$. This proves the assertion: a connecting point of $\operatorname{Div}_{T}^{1, m}(j)$ and $\operatorname{Div}_{T}^{1, m}(j+1)$ is

$$
\Gamma_{1} \cup\left(\bar{\Gamma}_{2}+l_{1}\right)=\left(\Gamma_{1}+l_{1}\right) \cup \bar{\Gamma}_{2}
$$

Next, we need the following:

Claim 6.17. Any $\left[\Gamma_{T}\right] \in \operatorname{Div}_{T}^{1, m}$ is connected and non-special, i.e. $h^{1}\left(\mathcal{O}_{\Gamma_{T}}(1)\right)=0$. Therefore

$$
h^{0}\left(\mathcal{O}_{\Gamma_{T}}(1)\right)=m-g+1 .
$$

Proof of Claim 6.17. Consider the exact sequence

$$
0 \rightarrow \mathcal{O}_{T}\left(H-\Gamma_{T}\right) \rightarrow \mathcal{O}_{T}(H) \rightarrow \mathcal{O}_{\Gamma_{T}}(H) \rightarrow 0 .
$$

From the definition of $T$, it follows that $h^{1}\left(\mathcal{O}_{T}(H)\right)=0$ because $T$ is linearly normal in $\mathbb{P}^{R}$.

To prove non-speciality of $\Gamma_{T}$, we will prove $h^{2}\left(\mathcal{O}_{T}\left(H-\Gamma_{T}\right)\right)=0$. We recall that $T$ is a connected union of two smooth, irreducible surfaces, with normal crossings, so the dualizing sheaf of $T$ is associated to a Cartier divisor, denoted by $K_{T}$; by Serre duality, we need to compute $h^{0}\left(\mathcal{O}_{T}\left(K_{T}-H+\Gamma_{T}\right)\right)$.

Since

$$
\mathcal{O}_{T}\left(K_{T}-H+\Gamma_{T}\right) \hookrightarrow \mathcal{O}_{X}\left(K_{T}-H+\Gamma_{T}\right) \oplus \mathcal{O}_{Q}\left(K_{T}-H+\Gamma_{T}\right),
$$

it suffices to prove $h^{0}\left(\left.\mathcal{O}_{T}\left(K_{T}-H+\Gamma_{T}\right)\right|_{\Sigma}\right)=0$, where $\Sigma$ is either $Q$ or $X$.

In the former case, if $l$ and $r$ denote the two distinct rulings on $Q$, we get

$$
h^{0}\left(\left.\mathcal{O}_{T}\left(K_{T}-H+\Gamma_{T}\right)\right|_{Q}\right)=h^{0}\left(\mathcal{O}_{Q}\left(\left(m-m_{X}-2\right) l-2 r\right)\right)=0,
$$

since $\left.H\right|_{Q} \sim l+r,\left.K_{T}\right|_{Q} \sim K_{Q}+l_{1}+l_{2}$ and $\left.\Gamma_{T}\right|_{Q} \sim r+\left(m-m_{X}-1\right) l$. With similar computations, if $f$ denotes the ruling of $X$, we obtain

$$
h^{0}\left(\left.\mathcal{O}_{T}\left(K_{T}-H+\Gamma_{T}\right)\right|_{X}\right)=h^{0}\left(\mathcal{O}_{X}(-2 H+(m+2 g-2) l)\right)=0,
$$

which proves non-speciality of $\Gamma_{T}$.

Since $\Gamma_{T}$ is an effective Cartier divisor on $T$, from the exact sequence

$$
0 \rightarrow \mathcal{O}_{T}\left(-\Gamma_{T}\right) \rightarrow \mathcal{O}_{T} \rightarrow \mathcal{O}_{\Gamma_{T}} \rightarrow 0
$$

and from analogous computations as above, one shows that $h^{1}\left(\mathcal{O}_{T}\left(-\Gamma_{T}\right)\right)=h^{1}\left(\mathcal{O}_{T}\left(K_{T}+\right.\right.$ $\left.\left.\Gamma_{T}\right)\right)=0$, so $h^{0}\left(\mathcal{O}_{\Gamma_{T}}\right)=h^{0}\left(\mathcal{O}_{T}\right)=1$. By Riemann-Roch theorem on $\Gamma_{T}$, this gives (6.18).

Let $h:=m-g$; from the Euler sequence restricted to $\Gamma_{T}$ and from Claim 6.17, we have $h^{1}\left(\mathcal{N}_{\Gamma_{T} / \mathbb{P}^{h}}\right)=0$.

From the inclusions $\Gamma_{T} \subset \mathbb{P}^{h} \subset \mathbb{P}^{R}$, we have the exact sequence:

$$
0 \rightarrow \mathcal{N}_{\Gamma_{T} / \mathbb{P}^{h}} \rightarrow \mathcal{N}_{\Gamma_{T} / \mathbb{P}^{R}} \rightarrow \bigoplus_{d-g+1-m} \mathcal{O}_{\Gamma_{T}}(H) \rightarrow 0
$$


which shows that also $h^{1}\left(\mathcal{N}_{\Gamma_{T} / \mathbb{P}^{R}}\right)=0$.

Observe that $\Gamma_{T}$ is l.c.i. in $T$ and that $T$ is l.c.i. in $\mathbb{P}^{R}$, i.e. $\Gamma_{T} \subset T$ and $T \subset \mathbb{P}^{R}$ are regular embeddings (cf. e.g. [30]). From [30, Proposition 4.5.3], the pair $\left(T, \Gamma_{T}\right)$ corresponds to a smooth point of the Flag-Hilbert scheme $F_{d, g, m}$ parametrizing pairs $\left(S, \Gamma_{S}\right)$, with $[S] \in \mathcal{H}_{d, g}$ and $\left[\Gamma_{S}\right] \in \operatorname{Div}_{S}^{1, m}$ (cf. [30, $\left.\S 4.5\right]$ ), since the obstructions are contained in

$$
H^{1}\left(\mathcal{N}_{\Gamma_{T} / \mathbb{P}^{R}}\right) \times_{H^{1}\left(\mathcal{N}_{T / \mathbb{R} R} \otimes \mathcal{O}_{\Gamma_{T}}\right)} H^{1}\left(\mathcal{N}_{T / \mathbb{P}^{R}}\right)=(0) .
$$

Let us consider the projection

$$
F_{d, g, m} \stackrel{\pi}{\longrightarrow} \mathcal{H}_{d, g}
$$

For any $[S] \in \mathcal{H}_{d, g}$, the fibre of $\pi$ over $[S]$ with its scheme structure, coincides with $\operatorname{Div}_{S}^{1, m}$. As we saw, the fibre over [T], i.e. $\operatorname{Div}_{T}^{1, m}$ as in (6.11), consists of smooth points of $F_{d, g, m}$. Hence, there is a non-empty, open Zariski subset $U \subset \mathcal{H}_{d, g}$ such that $\pi^{-1}(U)$ consists of smooth points of $F_{d, g, m}$. By generic smoothness, it follows that the fibre over the general point $[S] \in U$, which is $\operatorname{Div}_{S}^{1, m}$, is smooth.

We are left to show that $\operatorname{Div}_{S}^{1, m}$ is connected, for $d_{m}>0$ and for $[S] \in \mathcal{H}_{d, g}$ general. To this aim, consider the morphism $\pi$ as in (6.19). We need the following

Claim 6.20. $\operatorname{Div}_{T}^{1, m}$ is generically reduced, i.e. it is reduced at the general point of any irreducible component of dimension $d_{m}$.

Proof of Claim 6.20. To prove this, since $[T] \in \mathcal{H}_{d, g}$ is a smooth point, we have to show that if $\Gamma_{T}$ is a general point of a component of $\operatorname{Div}_{T}^{1, m}$, then the differential of the map $\pi$ at the point $\left(T, \Gamma_{T}\right)$ is surjective. From [30, $\left.\S 4.5\right]$, and from the diagram

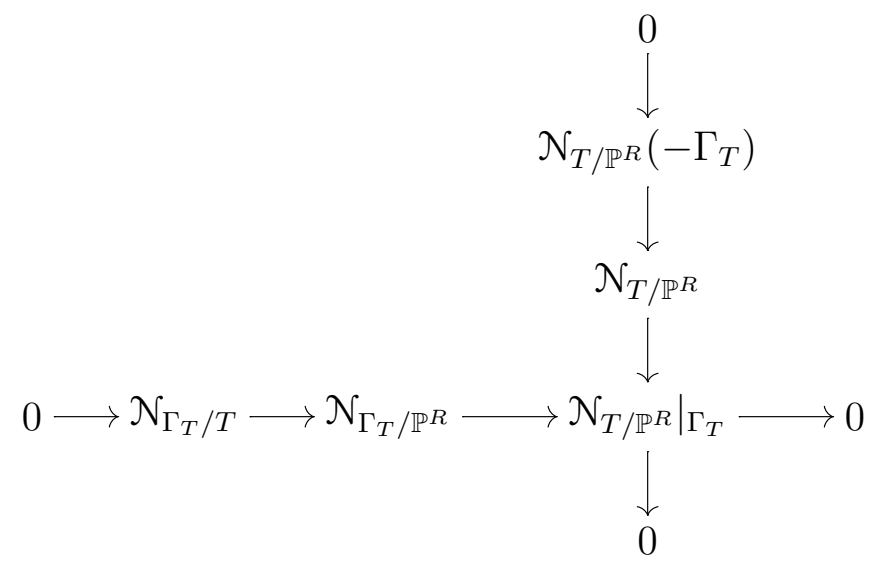

it suffices to show that $h^{1}\left(\mathcal{N}_{\Gamma_{T} / T}\right)=0$. We prove this by induction on $g$. Note that the case $g=0$ is trivially true.

A general point of an irreducible component of $\operatorname{Div}_{T}^{1, m}$ is a section $\Gamma_{T}$ of $T$, as in (6.10), from which we keep notation.

Since $\Gamma_{T}$ is a Cartier divisor, $\mathcal{N}_{\Gamma_{T} / T}=\mathcal{O}_{\Gamma_{T}}\left(\Gamma_{T}\right)$. Let $\nu: C \rightarrow \Gamma_{T}$ be the normalization morphism. Then, $C$ is the disjoint union of two smooth, irreducible curves $C=C_{X} \cup C_{Q}$, where $C_{X} \cong \Gamma_{X}$ and $C_{Q} \cong \Gamma_{Q}$. One has the standard exact sequence

$$
0 \rightarrow \mathcal{O}_{\Gamma_{T}} \rightarrow \nu_{*}\left(\mathcal{O}_{C}\right) \rightarrow \mathcal{O}_{\left\{p_{1}, p_{2}\right\}} \rightarrow 0
$$

If we tensor (6.21) with $\mathcal{O}_{\Gamma_{T}}\left(\Gamma_{T}\right)$, we get

$$
0 \rightarrow \mathcal{O}_{\Gamma_{T}}\left(\Gamma_{T}\right) \rightarrow \nu_{*}\left(\mathcal{O}_{C}\right) \otimes \mathcal{O}_{\Gamma_{T}}\left(\Gamma_{T}\right) \rightarrow \mathcal{O}_{\left\{p_{1}, p_{2}\right\}} \rightarrow 0 .
$$


Since $\nu$ is a finite morphism,

$$
H^{1}\left(\nu_{*}\left(\mathcal{O}_{C}\right) \otimes \mathcal{O}_{\Gamma_{T}}\left(\Gamma_{T}\right)\right) \cong H^{1}\left(\mathcal{O}_{C}\left(\nu^{*}\left(\Gamma_{T}\right)\right)\right) .
$$

Thus,

$$
H^{1}\left(\mathcal{O}_{C}\left(\nu^{*}\left(\Gamma_{T}\right)\right)\right) \cong H^{1}\left(\mathcal{O}_{C_{X}}\left(C_{X}\right)\right) \oplus H^{1}\left(\mathcal{O}_{C_{Q}}\left(C_{Q}\right)\right) \cong H^{1}\left(\mathcal{N}_{\Gamma_{X} / X}\right) \oplus H^{1}\left(\mathcal{N}_{\Gamma_{Q} / Q}\right)
$$

By induction, this is zero.

By (6.22), to prove $h^{1}\left(\mathcal{N}_{\Gamma_{T} / T}\right)=0$ it suffices to prove that the map

$$
H^{0}\left(\nu_{*}\left(\mathcal{O}_{C}\right) \otimes \mathcal{O}_{\Gamma_{T}}\left(\Gamma_{T}\right)\right) \stackrel{\rho}{\rightarrow} H^{0}\left(\mathcal{O}_{\left\{p_{1}, p_{2}\right\}}\right) \cong \mathbb{C}^{2}
$$

is surjective.

As above, $H^{0}\left(\mathcal{O}_{C}\left(\nu^{*}\left(\Gamma_{T}\right)\right)\right) \cong H^{0}\left(\mathcal{N}_{\Gamma_{X} / X}\right) \oplus H^{0}\left(\mathcal{N}_{\Gamma_{Q} / Q}\right)$ and the map $\rho$ is given by

$$
\rho\left(\left(\sigma_{1}, \sigma_{2}\right)\right)=\left(\sigma_{1}\left(p_{1}\right)-\sigma_{2}\left(p_{1}\right), \sigma_{1}\left(p_{2}\right)-\sigma_{2}\left(p_{2}\right)\right) .
$$

Since, by assumption, $d_{m}>0$, two cases have to be considered, as in the proof of Claim 6.13. If $m-m_{X}>1$, by the expression (6.24), $\rho$ is clearly surjective. If $m-m_{X}=1$, by induction $d_{m_{X}}=d_{m}+1 \geq 2$; thus, also in this case the map $\rho$ is surjective, because by the genericity of the lines $l_{1}$ and $l_{2}$ and of the quadric $Q$, the points $p_{1}$ and $p_{2}$ are general on $T$, hence they give independent conditions to the curves in $\operatorname{Div}_{X}^{1, m_{X}}$. This ends the proof of the claim.

We claim further that $\operatorname{Div}_{T}^{1, m}$ is actually reduced. Indeed, since $F_{d, g, m}$ is smooth along the fibre of $\pi$ over $[T]$, this fibre is locally complete intersection in $F_{d, g, m}$, hence it has no embedded component. Thus, being generically reduced and with no embedded component, this fibre, isomorphic to $\operatorname{Div}_{T}^{1, m}$, is reduced.

Since $\operatorname{Div}_{T}^{1, m}$ is connected, by Claim 6.16, and reduced, then $h^{0}\left(\mathcal{O}_{\operatorname{Div}_{T}^{1, m}}\right)=1$. Finally, the connectedness of $\operatorname{Div}_{S}^{1, m}$, for $[S] \in \mathcal{H}_{d, g}$ general, follows by the flatness of $\pi$ over $[T]$ and by semicontinuity.

Remark 6.25. The proof of Theorem 6.9 also shows that $\operatorname{Div}_{T}^{1, m}$ is the flat limit of $\operatorname{Div}_{S}^{1, m}$.

Remark 6.26. Observe that Theorem [6.9 implies that, if $[S] \in \mathcal{H}_{d, g}$ is general and $[\Gamma] \in$ $\operatorname{Div}_{S}^{1, m}$, then

$$
h^{1}\left(\mathcal{N}_{\Gamma / S}\right)=0
$$

Indeed, $h^{0}\left(\mathcal{N}_{\Gamma_{S} / S}\right)=d_{m}$ by smoothness and (6.27) follows by Riemann-Roch.

\section{Some EnUmerative RESUlts}

The aim of this section is to prove some enumerative results concerning the scheme $\operatorname{Div}_{S}^{1, m}$, for $[S] \in \mathcal{H}_{d, g}$ general, with $d$ and $g$ as in Theorem 5.3.

7.1. Ghione's theorem. In [11, Théorème 6.4 and 6.5] Ghione proves some basic enumerative properties concerning unisecant curves of a general ruled surfaces of degree $d$ and genus $g$, which were originally stated by C. Segre (cf. [29]). According to Theorem 6.7, Ghione's results are asymptotical, i.e. they apply to scrolls of sufficiently high degree $d$. Theorem 6.9 allows us to prove a more precise statement for ruled surfaces over a curve with general moduli.

Theorem 7.1. Let $g \geq 0$ and $d$ be as in Theorem [5.3. Let $[S] \in \mathcal{H}_{d, g}$ be the general point. Let $\bar{m}:=\left\lfloor\frac{d+g}{2}\right\rfloor$. Then the minimal degree of the unisecant curves of $S$ is

(1) $\frac{d+g-1}{2}$ if $d+g$ is odd; moreover there are $2^{g}$ such sections; 
(2) $\frac{d+g}{2}$ if $d+g$ is even; moreover there is a smooth, irreducible, one-dimensional family of such sections.

Proof. The case $g=0$ is well known: since $[S] \in \mathcal{H}_{d, 0}$ is general, then $S \subset \mathbb{P}^{d+1}$ is a smooth, balanced rational normal scroll of degree $d$ (cf. e.g. [3, Proposition 3.8]).

For $g \geq 1$, apply Theorems [5.4, 6.9 and [11, Théorème 6.4 and 6.5].

As a byproduct of the proof of Proposition 7.3 below, we will give an alternative proof of the enumerative part of Theorem 7.1 (cf. Remark 7.4).

7.2. The index computation. Let $g \geq 0$ and $d$ be as in Theorem $\left[5.3\right.$ and let $[S] \in \mathcal{H}_{d, g}$ be the general point. Let $m>\frac{d+g}{2}$ be an integer. Then, $\operatorname{dim}\left(\operatorname{Div}_{S}^{1, m}\right)=d_{m}>0$ (see (6.4) $)$.

The index of $\operatorname{Div}_{S}^{1, m}$ is the number of curves in $\operatorname{Div}_{S}^{1, m}$ passing through $d_{m}$ general points on $S$ (cf. e.g. [29, $\S 11$, p. 137]).

The following result (see [29, $\S 4$, p. 132, §12, 13, pp. 138-140]) can be derived from Theorem 7.1.

Proposition 7.2. Hypotheses as in Theorem 7.1. Then, the index of $\operatorname{Div}_{S}^{1, m}$ is $2^{g}$.

Proof. The case $g=0$ is trivial, so assume $g \geq 1$. Let $\Lambda$ be a set of $d_{m}$ general points on $S$ and let $\Gamma$ be any unisecant curve of $S$ of degree $m$ passing through $\Lambda$. Denote by $\pi_{\Lambda}: S \rightarrow \mathbb{P}^{R^{\prime}}$ the projection of $S \subset \mathbb{P}^{R}$ from $\Lambda$, where $R=d-2 g+1$.

If $S^{\prime}:=\pi_{\Lambda}(S)$, then $S^{\prime} \subset \mathbb{P}^{R^{\prime}}$ is a smooth, non-special scroll of genus $g^{\prime}=g$ such that:

- $d^{\prime}:=\operatorname{deg}\left(S^{\prime}\right)=d-d_{m}=2 d+g-2 m-1$,

- $R^{\prime}=R-d_{m}=2 d-2 m-g=d^{\prime}-2 g^{\prime}+1$.

The numerical assumptions of Theorem 5.3 hold for $S^{\prime}$. Furthermore, by generality of $[S] \in$ $\mathcal{H}_{d, g}$, also $\left[S^{\prime}\right] \in \mathcal{H}_{d^{\prime}, g^{\prime}}$ is a general point.

Denote by $\Gamma^{\prime}=\pi_{\Lambda}(\Gamma)$. Then $\left[\Gamma^{\prime}\right] \in \operatorname{Div}_{S^{\prime}}^{1, m^{\prime}}$, where $m^{\prime}=m-d_{m}=d+g-1-m$. Since

$$
d^{\prime}+g^{\prime}=d-d_{m}+g=2(d+g-m)-1
$$

is odd then, for any $d, g$ and $m$ as above, $\operatorname{dim}\left(\operatorname{Div}_{S^{\prime}}^{1, m^{\prime}}\right)=d_{m^{\prime}}=0$. Thus, by Theorem 7.1 , any such $\Gamma^{\prime}$ is a section of minimal degree of $S^{\prime}$ and there exist $2^{g^{\prime}}=2^{g}$ such sections.

One concludes by observing that the correspondence between elements of $\operatorname{Div}_{S}^{1, m}$ passing through $\Lambda$ and elements of $\operatorname{Div}_{S^{\prime}}^{1, m^{\prime}}$ is bijective.

7.3. The monodromy action. In this subsection, we study the monodromy of the $2^{g}$ sections of minimal degree $\bar{m}$ of a scroll $S$ corresponding to the general point of $\mathcal{H}_{d, g}$, when $d_{\bar{m}}=0$.

Proposition 7.3. Let $g \geq 1, d$ and $\mathcal{H}_{d, g}$ be as in Theorem 5.3. Assume $d+g$ odd and let $\bar{m}=\frac{d+g-1}{2}$. Then, for $[S] \in \mathcal{H}_{d, g}$ general, the monodromy acts on $\operatorname{Div}_{S}^{1, \bar{m}}$ as the full symmetric group.

Proof. Let $[Y] \in \mathcal{H}_{d, g}$ be as in Construction 5.11. As in the proof of Theorem 6.9, a unisecant curve $\Gamma_{Y}$ of $Y$ will be a union of a unisecant curve $\Gamma_{W}$ of $W$ and unisecant curves $\Gamma_{j}$ of the quadrics $Q_{j}$, for any $1 \leq j \leq g$, i.e.

$$
\Gamma_{Y}=\Gamma_{W} \cup\left(\bigcup_{i=1}^{g} \Gamma_{j}\right),
$$

with matching conditions on the rulings $l_{1, j}, l_{2, j}$, for $1 \leq j \leq g$. 
Since we want to consider unisecant curves of $S$, properly contained in its hyperplane section, it immediately follows that any $\Gamma_{j}$ on $Q_{j}$ is either a conic, say $C_{j}$, or a line, say $r_{j}$, not belonging to the ruling $\left|l_{i, j}\right|$, for $1 \leq i \leq 2$ and $1 \leq j \leq g$.

Up to a permutation of the indices, for some $0 \leq k \leq g$, we may assume we have conics

$$
C_{j} \subset Q_{j}, \text { for } 1 \leq j \leq k \leq g
$$

and lines

$$
r_{j} \subset Q_{j}, \text { for } j>k,
$$

when $k \geq 1$, or only lines, when $k=0$.

In any case,

$$
\Gamma_{Y}=\Gamma_{W} \cup\left(\bigcup_{j \leq k} C_{j}\right) \cup\left(\bigcup_{i=k+1}^{g} r_{i}\right),
$$

therefore

$$
\operatorname{deg}\left(\Gamma_{W}\right):=\nu_{k}=\frac{d+g-1}{2}-2 k-(g-k)=\frac{d-g-1}{2}-k .
$$

Notice that one has $\nu_{k} \geq \frac{d-2 g-1}{2}$ which, by Theorem 7.1 , is the minimal degree for the unisecant curves on $W$; hence $g \geq 2 k$. Moreover, the unisecant curves $\Gamma_{W}$ form a complete linear system $\Lambda_{k}$ on $W$ and, by (6.4), one has

$$
\operatorname{dim}\left(\Lambda_{k}\right)=g-2 k
$$

Consider

$$
\bigcup_{1 \leq i \leq 2,1 \leq j \leq g} l_{i, j} \cong\left(\mathbb{P}^{1}\right)^{2 g}
$$

For any $k \geq 0$, we have a rational map

$$
\lambda_{k}: \Lambda_{k} \rightarrow\left(\mathbb{P}^{1}\right)^{2 g},
$$

which is generically injective. Let $V_{k}:=\operatorname{Im}\left(\lambda_{k}\right)$; then $\left[V_{k}\right]$ is a cycle of dimension $g-2 k$ in the Chow ring of $\left(\mathbb{P}^{1}\right)^{2 g}$.

Consider the projection to the $i$-th factor

$$
\left(\mathbb{P}^{1}\right)^{2 g}=\left(\mathbb{P}^{1} \times \mathbb{P}^{1}\right)^{g} \stackrel{\pi_{i}}{\longrightarrow}\left(\mathbb{P}^{1} \times \mathbb{P}^{1}\right)_{i} .
$$

Set

$$
H_{i}=\pi_{i}^{*}\left(\mathcal{O}_{\mathbb{P}^{1} \times \mathbb{P}^{1}}(1)\right), 1 \leq i \leq g .
$$

Thus, $\left|H_{i}\right|$ is base-point-free, for any $1 \leq i \leq g$. Therefore,

$$
\operatorname{dim}\left(V_{k} \cap\left(\bigcap_{t=k+1}^{g} H_{t}\right)\right)=\max \{-1,-k\},
$$

where $H_{i}$ is general in $\left|H_{i}\right|$, for $k+1 \leq i \leq g$. By generality and by the matching conditions, we need this dimension to be non-negative. This implies $k=0$.

In the above setting, define $V_{0, i}:=V_{0} \cap H_{1} \cap \ldots \cap H_{i}$ for $i \geq 0$; with this notation $V_{0,0}$ coincides with $V_{0}$. One has

$$
v_{0, i}:=\operatorname{dim}\left(V_{0, i}\right)=\max \{-1, g-2 k-i\} .
$$

We claim that $V_{0, i}$ is irreducible as soon as $v_{0, i} \geq 1$, i.e. $g \geq 2 k+i+1$. The assertion holds for $V_{0,0}=V_{0}$, so we proceed by induction on $i$. Thus we assume $i \geq 1, v_{0, i} \geq 1$ and $V_{0, i-1}$ irreducible of dimension $v_{0, i-1}=v_{0, i}+1 \geq 2$. Let us prove that the projection $\pi_{i}^{\prime}: V_{0, i-1} \rightarrow\left(\mathbb{P}^{1} \times \mathbb{P}^{1}\right)_{i}$ is dominant. To see this, let $\Lambda_{0, i-1}$ be the pull-back via the map $\lambda_{0}$ of $V_{0, i-1}$. This is a sublinear system of $\Lambda_{0}$ of dimension $v_{0, i}+1 \geq 2$. If $\pi_{i}^{\prime}$ were not dominant, 
then, by our generality assumptions, the linear system $\Lambda_{0, i-1}$ of unisecants would map two general lines of the scroll $W$ to the same line, hence $\Lambda_{0, i-1}$ would be a pencil, a contradiction. Since $\pi_{i}^{\prime}$ is dominant, the claim follows by Bertini's theorem.

Finally, $V_{0, g-1}$ is an irreducible curve and $\Lambda_{0, g-1}$ is a pencil. The same argument as above shows that $\pi_{g}$ maps $V_{0, g-1}$ injectively to an irreducible curve $\Gamma$ on the smooth quadric $Q_{g} \cong$ $\left(\mathbb{P}^{1} \times \mathbb{P}^{1}\right)_{g} \subset \mathbb{P}^{3}$. By [1, Lemma, p. 111], the monodromy group of this curve in $\mathbb{P}^{3}$ is the full symmetric group, implying the assertion.

Remark 7.4. With the same ideas as in the proof of Proposition 7.3 , we can give an alternative proof of the fact that, when $d+g$ is odd, then $S$ contains $2^{g}$ sections of minimal degree. Indeed, since $[Y] \in \mathcal{H}_{d, g}$ is a smooth point and the map $\pi$ as in (6.19) is in this case smooth over $[Y]$ (see the proof of Theorem 6.9) , we see that $\operatorname{deg}\left(\operatorname{Div}_{S}^{1, \frac{d+g-1}{2}}\right)=\operatorname{deg}(\Gamma)$, where $\Gamma$ is the curve on the smooth quadric $Q_{g}$ considered at the end of Proposition 7.3 .

It is not difficult to see that $\operatorname{deg}(\Gamma)=2^{g}$. In fact, any $H_{i} \subset\left(\mathbb{P}^{1}\right)^{2 g}$ is linearly equivalent to $r_{1, i}+r_{2, i}$, where $r_{j, i}, 1 \leq j \leq 2$, are the pull-backs, via $\pi_{i}$, of the two rulings of $\left(\mathbb{P}^{1} \times \mathbb{P}^{1}\right)_{i}$. Therefore, in the Chow ring of $\left(\mathbb{P}^{1}\right)^{2 g}$, one has

$$
H_{1} \cdot H_{2} \cdot \ldots \cdot H_{g}=\sum_{\underline{i}, \underline{j}} R_{\underline{i}, \underline{j}}
$$

where $R_{\underline{i}, \underline{j}}:=r_{1, i_{1}} \ldots r_{1, i_{k}} r_{2, j_{1}} \ldots r_{2, j_{h}}$ and $i_{1}<i_{2}<\cdots<i_{k}, j_{1}<j_{2}<\cdots<j_{h}$, such that $\left\{i_{1}, i_{2}, \cdots, i_{k}, j_{1}, j_{2}, \cdots, j_{h}\right\}=\{1, \cdots, g\}$, in particular $h+k=g$. For any summand, one has

$$
R_{\underline{i}, \underline{j}} \cdot V_{0}=1 .
$$

The intersection is in fact equivalent to imposing to the linear system $\Lambda_{0}$, of dimension $g$, $g$ general points on $W$. The assertion follows since the right hand side of (7.5) contains $2^{g}$ summands.

7.4. The genus computation. Let $g \geq 0$ and $d$ be as in Theorem $\left[5.3\right.$ and let $[S] \in \mathcal{H}_{d, g}$ be the general point. Let $m>\frac{d+g}{2}$ be an integer. Then, $\operatorname{dim}\left(\operatorname{Div}_{S}^{1, m}\right)=d_{m}>0($ see (6.4) $)$.

Given $Z$ a general 0-dimensional subscheme of $S$ of length $d_{m}-1$, there is a 1-dimensional family $\mathcal{D} \subset \operatorname{Div}_{S}^{1, m}$ consisting of curves containing $Z$. The following proposition computes the genus of $\mathcal{D}$, slightly extending the results in [16] and [27, Example 3.2].

Proposition 7.6. Hypotheses as in Theorem 5.3. Then, $\mathcal{D}$ is smooth and irreducible, of genus

$$
\gamma:=2^{g}(g-1)+1
$$

Proof. We prove the assertion in case $d_{m}=1$. The case $d_{m}>1$ can be dealt with as in Proposition 7.2 .

As in the proof of Proposition 7.3 , we can consider a cycle $V_{k}^{\prime}, 1 \leq k \leq g$, in the Chow-ring of $\left(\mathbb{P}^{1}\right)^{2 g}$, which is the image of the complete linear system $\Lambda_{k}^{\prime}$ on $W$ of unisecant curves of degree

$$
\mu_{k}:=\frac{d+g}{2}-2 k-(g-k)=\frac{d-g}{2}-k
$$

via the obvious rational map $\lambda_{k}^{\prime}: \Lambda_{k}^{\prime}-\rightarrow\left(\mathbb{P}^{1}\right)^{2 g}$. Then $\operatorname{dim}\left(\Lambda_{k}^{\prime}\right)=\operatorname{dim}\left(V_{k}^{\prime}\right)=g+1-2 k$ and

$$
\operatorname{dim}\left(V_{k}^{\prime} \cap\left(\bigcap_{t=k+1}^{g} H_{t}\right)\right)=\max \{-1,1-k\} .
$$


In the present situation, we need the above dimension to be either 0 or 1 , i.e. either $k=0$ or $k=1$.

(1) If $k=0, \operatorname{dim}\left(V_{0}^{\prime}\right)=g+1$ and the curve

$$
\Xi_{0}:=V_{0}^{\prime} \cap\left(\bigcap_{j=1}^{g} H_{j}\right),
$$

which is smooth and irreducible (see proof of Proposition [7.3), is a component of the family of unisecant curves on $Y$.

(2) If $k=1$, on $W$ we have the linear system $\Lambda_{1}^{\prime}$ of curves of degree $\mu_{1}=\frac{d-g-2}{2}$, where $\operatorname{dim}\left(\Lambda_{1}^{\prime}\right)=g-1$, and there exists an index $l \in\{1, \ldots, g\}$ such that on the quadric $Q_{l}$ we have conics, whereas on the quadrics $Q_{j}$, for $1 \leq j \neq l \leq g$, we have lines. In this case, for any $1 \leq l \leq g$, we have a reduced, 0 -dimensional scheme

$$
\Xi_{1, l}:=V_{1}^{\prime} \cap\left(\bigcap_{1 \leq j \neq l \leq g} H_{j}\right) .
$$

For each point in $\Xi_{1, l}$ we have a rational component of the family of unisecant curves on $Y$.

First we compute the class of $\Xi_{0}$. As in Remark 7.4,

$$
H_{1} \cdot H_{2} \cdot \ldots \cdot H_{g}=\sum_{\underline{i}, \underline{j}} R_{\underline{i}, \underline{j}} .
$$

Fix general points $p_{i} \in l_{1, i}$ and $q_{i} \in l_{2, i}$. Consider $\underline{i}=\left(i_{1}, \ldots, i_{k}\right)$ and $\underline{j}=\left(j_{1} \ldots, j_{h}\right)$ such that $\left\{i_{1}, \ldots, i_{k}, j_{1}, \ldots, j_{h}\right\}=\{1, \ldots, g\}$ and let $\Lambda_{0, \underline{i}, \underline{j}}^{\prime}$ be the sublinear system of $\Lambda_{0}^{\prime}$ consisting of all curves containing $p_{i_{1}}, \ldots, p_{i_{k}}, q_{j_{1}}, \ldots, q_{j_{h}}$. This is a pencil, whose image $\Xi_{\underline{i}, j}$ in $\left(\mathbb{P}^{1}\right)^{2 g}$ has class $R_{\underline{i}, \underline{j}} \cdot V_{0}^{\prime}$. Hence $\Xi_{0}$ is homologous to $\sum_{\underline{i}, \underline{j}} \Xi_{\underline{i}, \underline{j}}$, i.e. to a sum of $2^{g}$ copies of $\mathbb{P}^{1}$. It is not difficult to see how they intersect each other. Indeed $\Xi_{\underline{i}, \underline{j}} \cdot \Xi_{\underline{i}^{\prime}, \underline{j}^{\prime}}$ is non-zero, and it is a point, if and only if $\left\{p_{i_{1}}, \ldots, p_{i_{k}}, q_{j_{1}}, \ldots, q_{j_{h}}\right\} \cap\left\{p_{i_{1}}, \ldots, p_{i_{k}^{\prime}}, q_{j_{1}}, \ldots, q_{j_{h}^{\prime}}\right\}$ consists of exactly $g-1$ points. This implies that each $\Xi_{\underline{i}, \underline{j}}$ intersects exactly $g$ others $\Xi_{\underline{i}^{\prime}, \underline{j}^{\prime}}$.

Fix now $l$ any integer such that $1 \leq l \leq g$. Let $\underline{a}$ (resp., $\underline{b}$ ) denote a sequence of integers $a_{1}<a_{2}<\cdots<a_{k}$ (resp., $b_{1}<b_{2}<\cdots<b_{h}$ ), such that $h+k=g-1$ and $\left\{a_{1}, a_{2} \ldots, a_{k}, b_{1}, b_{2}, \ldots, b_{h}\right\}=\{1, \ldots, g\} \backslash\{l\}$. Let $D_{l, \underline{a}, \underline{b}}$ be the unique curve of $\Lambda_{1}^{\prime}$ containing $p_{i_{1}}, \ldots, p_{i_{k}}, q_{j_{1}}, \ldots, q_{j_{h}}$. This matches a pencil of conics on the quadric $Q_{l}$, to give a smooth rational component $\Xi_{l, \underline{a}, \underline{b}}^{\prime}$ of the family of unisecant curves on $Y$ as described in (2). These rational curves do not intersect each other. However, they intersect the curves $\Xi_{\underline{i}, \underline{j}}$, and precisely only two of them, i.e. the ones for which either $\underline{i}$ consists of $\underline{a}$ and $l$, or $\underline{j}$ consists of $\underline{b}$ and $l$.

As in the proof of Theorem 6.9, we see that the flat limit of the smooth curve $\mathcal{D}=\operatorname{Div}_{S}, \frac{d+g}{2}$ on $[S] \in \mathcal{H}_{d, g}$ general can be in turn flatly degenerated to a union of smooth rational curves whose dual graph $G$ has:

- $v=2^{g}+2^{g-1} \cdot g=2^{g-1}(2+g)$ vertices and

- $e=g \cdot \frac{2^{g}}{2}+2 \cdot 2^{g-1} \cdot g=3 \cdot 2^{g-1} \cdot g$ edges.

Hence $\chi(G)=v-e=2^{g}(1-g)$ and therefore the arithmetic genus of $\mathcal{D}$ is $\gamma=1-\chi(G)=$ $1+2^{g}(g-1)$. 
Notice that, when $g=2$, then $\gamma=5$. This also follows, via a different approach, from [27, Remark 1.6], because in this case the curve $\operatorname{Div}_{S}^{1, \frac{d+2}{2}}$ is isomorphic to a divisor in $|2 \Theta|$ in the jacobian of the curve $C$.

\section{REFERENCES}

[1] E. Arbarello, M. Cornalba, P.A. Griffiths, J. Harris, Geometry of algebraic curves, Vol. I. Grundlehren der Mathematischen Wissenschaften, 267, Springer-Verlag, New York, 1985.

[2] E. Arrondo, M. Pedreira, I. Sols, On regular and stable ruled surfaces in $\mathbb{P}^{3}$, Algebraic curves and projective geometry (Trento, 1988), 1-15. With an appendix of R. Hernandez, 16-18, Lecture Notes in Math., 1389, Springer-Verlag, Berlin, 1989.

[3] A. Calabri, C. Ciliberto, F. Flamini, R. Miranda, Degenerations of scrolls to unions of planes, Rend. Lincei Mat. Appl., 17 (2006), no. 2, 95-123.

[4] A. Calabri, C. Ciliberto, F. Flamini, R. Miranda, Brill-Noether theory and non-special scrolls, preprint, (2007).

[5] G. Castelnuovo, Sui multipli di una serie lineare di gruppi di punti appartenenti ad una curva algebrica, Rend. Circ. Mat. Palermo, 7 (1893), no. 3, 99-119.

[6] C. Ciliberto, A.F. Lopez, R. Miranda, Projective degenerations of $K 3$ surfaces, Gaussian maps, and Fano threefolds, Invent. Math., 114 (1993), no. 3, 641-667.

[7] C. Ciliberto, A.F. Lopez, R. Miranda, Some remarks on the obstructedness of cones over curves of low genus, Higher dimensional complex varieties, Proceedings of the Trento conference (1994), 167-182, De Gruyter, Berlin, 1996.

[8] L. Fuentes-Garcia, M. Pedreira, Canonical geometrically ruled surfaces, Math. Nachr., 278 (2005), no. $3,240-257$.

[9] L. Fuentes-Garcia, M. Pedreira, The projective theory of ruled surfaces, Note Mat., 24 (2005), no. $1,25-63$.

[10] L. Fuentes-Garcia, M. Pedreira, The general special scroll of genus $g$ in $\mathbb{P}^{N}$. Special scrolls in $\mathbb{P}^{3}$, math.AG/0609548 (2006), pp. 13.

[11] F. Ghione, Quelques résultats de Corrado Segre sur les surfaces réglées, Math. Ann., 255 (1981), $77-95$.

[12] F. Ghione, G. Sacchiero, Genre d'une courbe lisse tracée sur une variété réglée, Space curves (Rocca di Papa, 1985), 97-107, Lecture Notes in Math., 1266, Springer-Verlag, Berlin, 1987.

[13] L. Giraldo, I. Sols, The irregularity of ruled surfaces in $\mathbb{P}^{3}$. Dedicated to the memory of Fernando Serrano, Collect Math., 49 (1998), no. 2-3, 325-334.

[14] S. Greco, Normal varieties. Notes written with the collaboration of A. Di Sante, Inst. Math., 4, Academic Press, London, 1978.

[15] P. Griffiths, J. Harris, Principles of Algebraic Geometry, Wiley Classics Library, New York, 1978.

[16] M.J. Gronow, Ph.D. thesis, University of Durham, 1997, unpublished.

[17] A. Grothendieck, Technique de descente et théorème d'existence en géométrie algébrique. V Séminaire Bourbaki, 232, 1961-1962.

[18] Hartshorne R., Algebraic Geometry, Graduate Texts in Math., 52, Springer-Verlag, New York, 1977.

[19] Huybrechts D., Lehn M., The Geometry of Moduli spaces of sheaves, Aspects of Mathematics, E31. Friedr. Vieweg \& Sohn, Braunschweig, 1997.

[20] A. Lanteri, C. Turrini, On the automorphisms of the elliptic ruled surfaces, Boll. Un. Mat. Ital. (6), 1 (1982), no. 1, 41-58.

[21] A. Maruyama, On classification of ruled surfaces, Lectures in Mathematics. Kyoto University, no. 3, Tokyo, 1970.

[22] A. Maruyama, M. Nagata, Note on the structure of a ruled surface, J. reine angew. Math., 239 (1969), 68-73.

[23] M. Mattuck, Symmetric products and Jacobians, Am. J. Math., 83 (1961), 189-206.

[24] D. Mumford, Varieties defined by quadratic equations, in Questions on Algebraic Varieties - Corso CIME 1969, Rome (1970), 30-100.

[25] M. Nagata, On self-intersection number of a section on a ruled surface, Nagoya Math. J., 37 (1970), 191-196. 
[26] P.E. Newstead, Introduction to moduli problems and orbit spaces, Tata Institute of Fundamental Research Lectures on Mathematics and Physics, 51, Narosa Publishing House, New Delhi, 1978.

[27] W.M. Oxbury, Varieties of maximal line bundles, Math. Proc. Camb. Phil. Soc., 129 (2000), 9-18.

[28] C. Okonek, M. Schneider, H. Spindler, Vector bundles on complex projective spaces, Progress in Mathematics, 3, Birkhäuser, Boston, Mass., 1980.

[29] C. Segre, Recherches générales sur les courbes et les surfaces réglées algébriques, OPERE - a cura dell'Unione Matematica Italiana e col contributo del Consiglio Nazionale delle Ricerche, vol. 1, §XI - pp. 125-151, Edizioni Cremonese, Roma, 1957 (cf. Math. Ann., 34 (1889), 1-25).

[30] E. Sernesi, Deformations of Algebraic Schemes, Grundlehren der mathematischen Wissenschaften, 334, Springer-Verlag, Berlin, 2006.

[31] C.S. Seshadri, Fibrés vectoriels sur les courbes algébriques, Astérisque, 96, S.M.F., Paris, 1982.

[32] F. Severi, Vorlesungen ueber algebraische Geometrie, Teubner, Leipzig, 1921.

[33] F. Severi, Sulla classificazione delle rigate algebriche, Univ. Roma e Ist. Naz. Alta Mat. Rend. Mat. e Appl., 2 (1941), 1-32.

[34] L.W. Tu, Semistable bundles over an elliptic curve, Adv. Math., 98 (1993), no. 1, 1-26.

[35] G. Zappa, Sulla degenerazione delle superficie algebriche in sistemi di piani distinti, con applicazioni allo studio delle rigate, Atti R. Accad. d'Italia, Mem. Cl. Sci. FF., MM. e NN., 13 (2) (1943), 989-1021.

E-mail address: calabri@dmsa.unipd.it

Current address: Dipartimento di Metodi e Modelli Matematici per le Scienze Applicate, Università degli Studi di Padova, Via Trieste, 63 - 35121 Padova, Italy

E-mail address: cilibert@mat.uniroma2.it

Current address: Dipartimento di Matematica, Università degli Studi di Roma Tor Vergata, Via della Ricerca Scientifica - 00133 Roma, Italy

E-mail address: flamini@mat.uniroma2.it

Current address: Dipartimento di Matematica, Università degli Studi di Roma Tor Vergata, Via della Ricerca Scientifica - 00133 Roma, Italy

E-mail address: Rick.Miranda@ColoState.Edu

Current address: Department of Mathematics, 101 Weber Building, Colorado State University, Fort Collins, CO 80523-1874, USA 